\title{
Stormy water on Mars: the distribution and saturation of atmospheric water during the dusty season
}

Anna A. Fedorova ${ }^{1 * \dagger}$, Franck Montmessin ${ }^{2 \dagger}$, Oleg Korablev $^{1 \dagger}$, Mikhail Luginin ${ }^{1}$, Alexander Trokhimovskiy $^{1}$, Denis A. Belyaev ${ }^{1}$, Nikolay I. Ignatiev ${ }^{1}$, Franck Lefèvre ${ }^{2}$, Juan Alday ${ }^{3}$, Patrick G.J. Irwin ${ }^{3}$, Kevin S. Olsen ${ }^{2,3}$, Jean-Loup Bertaux ${ }^{1,2}$, Ehouarn Millour ${ }^{4}$, Anni Määttänen ${ }^{2}$, Alexey Shakun ${ }^{1}$, Alexey V. Grigoriev ${ }^{1,5}$, Andrey Patrakeev ${ }^{1}$, Svyatoslav Korsa ${ }^{1}$, Nikita Kokonkov ${ }^{1}$, Lucio Baggio ${ }^{2}$, Francois Forget ${ }^{4}$, Colin F. Wilson ${ }^{3}$

${ }^{1}$ Space Research Institute of the Russian Academy of Sciences (IKI RAS), Moscow, Russia

${ }^{2}$ Laboratoire Atmosphères Milieux Observations Spatiales (LATMOS), Université Paris-Saclay, Sorbonne Université, Centre National de la Recherche Scientifique, Guyancourt, France

${ }^{3}$ Physics Department, Oxford University, Oxford, United Kingdom

${ }^{4}$ Laboratoire de Météorologie Dynamique, Sorbonne Université, Centre National de la Recherche Scientifique, Jussieu, Paris, France

${ }^{5}$ Australian National University, Research School of Astronomy and Astrophysics, Mount Stromlo Observatory, Advanced Instrumentation and Technology Centre, Canberra, Australia

*Corresponding author. Email: fedorova@iki.rssi.ru

${ }^{\dagger}$ These authors contributed equally to this work

Abstract: The loss of water from Mars to space is thought to result from the transport of water to the upper atmosphere where it is disassociated to hydrogen, which escapes. Recent observations have suggested large rapid seasonal intrusions of water into the upper 
atmosphere, boosting the hydrogen abundance. We use the Atmospheric Chemistry Suite on the ExoMars Trace Gas Orbiter to characterize the water distribution with altitude. Water profiles during the 2018-2019 southern spring and summer stormy seasons show that high altitude water is preferentially supplied close to perihelion and supersaturation occurs even when clouds are present. This implies that the water potential to escape from Mars is stronger than previously thought.

One Sentence Summary: Widespread supersaturation of water vapor on Mars enhances its propagation to high altitudes and escape to space.

Mars once harbored an active hydrological cycle, as demonstrated by geological features on its surface, but no longer holds the quantity of water required to produce such geological imprints $(1,2)$. The planet's bulk inventory of water amounts to a Global Equivalent Layer (GEL) of 30 meters, mostly in its polar ice caps (2). That is more than 10 times smaller than the estimated amount of water that once flowed on the surface (1). Mars' enhanced concentration of heavy water HDO ( $\geq 5$ times larger than the terrestrial standard) (3-5), strengthens the hypothesis that the majority of Mars’ primordial water has escaped over time.

Water in the atmosphere is a negligible component of the planet's total water inventory, being equivalent to a global layer of $10 \mu \mathrm{m}$ thick, but nevertheless regulates the dissipation of water over time. Most Martian water has been lost to space because its decomposition products (atomic hydrogen and oxygen) reach the upper atmosphere where they can acquire sufficient thermal energy to overcome Mars' low gravity (a third of Earth's). Water decomposition is theorized to follow a complex reaction chain involving the recombination of $\mathrm{H}$ atoms into $\mathrm{H}_{2}$ on a timescale of centuries (6-8), buffering any short-term hydrogen abundance variations. This mechanism has been challenged by observations showing that freshly produced $\mathrm{H}$ atoms can reach the exosphere (the uppermost layer where the 
atmosphere thins out and exchanges matter with interplanetary space) on a monthly timescale $(9,10)$. The observed short-term variability of the hydrogen atoms populating the exosphere could be caused by direct deposition of water molecules at altitudes high enough to expose them to sunlight, which subsequently triggers a rapid enhancement of hydrogen atoms in the exosphere (11-13).

Testing this hypothesis requires characterizing the mechanisms contributing to upward water propagation through large-scale atmospheric circulation. One such mechanism is the cold trap imposed, like on the Earth, by water ice cloud formation at low altitude subsequent to water condensation. The latter is predicted to occur whenever water vapor partial exceeds saturation. Because of the vapor pressure law, the cold trap efficiency depends heavily on temperature and eventually limits the amount of water that can be transported to higher altitudes (14-16).

We investigate these processes using occultations of the Sun by the Martian atmosphere (henceforth solar occultations) where the vertical distributions of gases and particles can be directly observed. We used the Atmospheric Chemistry Suite (ACS) (17) on the ExoMars Trace Gas Orbiter (TGO) spacecraft. ACS is an assembly of three infrared spectrometers that together provide continuous spectral coverage from 0.7 to $17 \mu \mathrm{m}$, with a spectral resolving power ranging from 10,000 to 50,000 . Our dataset has been assembled by performing solar occultations with the near-infrared (NIR), mid-infrared (MIR) and thermal infrared in honor of Prof. V. I. Moroz (TIRVIM) channels of ACS. The NIR channel $(0.7-1.7 \mu \mathrm{m})$ encompasses absorption bands of $\mathrm{CO}_{2}, \mathrm{H}_{2} \mathrm{O}, \mathrm{CO}$ and $\mathrm{O}_{2}$ diagnostic of their molecular concentrations over altitudes of 5 to $100 \mathrm{~km}$ with a vertical resolution of 1 to $3 \mathrm{~km}$. TIRVIM (2-17 $\mu \mathrm{m})$ provides simultaneous information on dust and water ice particle abundance.

We retrieved the volume fraction of water (i.e. its mixing ratio) and temperature using established methods (18-20) including the joint extraction of $\mathrm{CO}_{2}$ and $\mathrm{H}_{2} \mathrm{O}$ molecular 
abundances from respectively the 1.57 and $1.38 \mu \mathrm{m}$ absorption bands. Spectra were fitted with a spectroscopic model at all altitudes below $100 \mathrm{~km}$, and the profiles of gaseous components were subsequently retrieved using an iterative algorithm (21). Figure 1A shows an example of model outputs fitted to the spectra, along with the resulting water vapor profile with altitude. The sensitivity to $\mathrm{H}_{2} \mathrm{O}$ depends on altitude as it is a strong function of both the total number of molecules along the line of sight and the signal-to-noise ratio (SNR). SNR decreases exponentially with increasing atmospheric opacity along the line of sight, which is dominated by suspended dust aerosols and icy particles. Sensitivity to water vapor reaches 0.1 parts per million by volume (ppmv) at low altitudes in clear atmospheric conditions and is typically better than 1 ppmv between 10 and $75 \mathrm{~km}$, rising up to 20 ppmv at $100 \mathrm{~km}(17$, 21).

ACS NIR resolves the spectral structure of the $\mathrm{CO}_{2}$ rotational band, providing simultaneous temperature and pressure parameters self-consistently (21). This simultaneity allows us to evaluate the local water vapor saturation state (Figure 1B), a necessary parameter to estimate how much water can pass through the condensation level and reach the upper atmosphere. For most occultations, the NIR water vapor and temperature profiles can also be evaluated against aerosol profiles from ACS TIRVIM or ACS MIR data (Figure 1B and S1; (21)).

The TGO Sun-synchronous near-polar orbit allows us to survey water vapor and aerosol vertical distributions on a global scale. TGO performs two occultations on each 2hour orbit. On average ACS NIR accomplished nine occultation observations per sol (Martian day) in both hemispheres (except during five periods of about 15 to 20 days each around $L_{s}(22) 175,205,270,330,355^{\circ}$, producing a dataset of $\sim 1700$ occultations between April $2018\left(L_{s} 163^{\circ} \mathrm{MY} 34\right.$, where MY is Martian Year) and March 2019 ( $\left.L_{s} 356^{\circ} \mathrm{MY} 34\right)$. A summary of our results is shown in Figure 2. 
During MY 34 southern spring and summer, the atmosphere was affected by two largescale dust storms; the first, storm 2018A ( $L_{s} 188^{\circ}-250^{\circ}$, (23)), enshrouded the planet globally (a Global Dust Storm, GDS), and the second, a recurrent large regional storm named C (24, $25)$, occurred later in the southern summer $\left(L_{s} 320^{\circ}-335^{\circ}\right)$.

Several prominent features are visible in Figure 2. Both hemispheres exhibit relatively high water vapor mixing ratios (vmr) throughout the southern spring-summer season. The southern hemisphere is distinctly wetter with vmr exceeding 50 ppmv in the $50-100 \mathrm{~km}$ altitude range, while above $40-50 \mathrm{~km}$ the northern hemisphere exhibits a gradual decline in vmr after $L_{s} 210^{\circ}$. Water vapor propagates recurrently up to $100 \mathrm{~km}$ in the south, around $L_{s}$ $200^{\circ}$ (during the GDS), $270^{\circ}$ and $290^{\circ}$. In the north, this only occurred during the GDS (see also Figure S2, (21)). This migration of water vapor to high altitudes does not correlate with an increase in temperature, as evident around $L_{s} 195^{\circ}$ in the north where water vapor propagates higher than altitudes where the temperature rises. In the southern hemisphere, this is also evident around perihelion, when Mars orbits closest to the Sun, which implies that water vapor at high altitudes is primarily controlled by the large-scale motion of the Martian atmosphere, particularly the upward branch of the cross-hemispheric atmospheric circulation cell, also known as Hadley cell.

The saturation state in each hemisphere is shown in Figure 2D, along with the temperature, water vapor, and aerosols. Large saturation ratios (1 to $>10)$ are present in both hemispheres below $30 \mathrm{~km}$ before and during the early phase of the GDS $\left(L_{s}<190^{\circ}\right)$. ACS was observing the latitudes poleward of $60^{\circ}$ at that time, implying that at least a third of the global atmosphere between 5 and $30 \mathrm{~km}$ was supersaturated. This is reminiscent of a previouslyobserved supersaturation phenomenon (19) yet extends over a greater vertical range. Similar features can be seen in both hemispheres during this period - thick water ice clouds in a supersaturated atmosphere - suggesting cloud formation does not impose the atmosphere to 
remain at saturation even when dust aerosols, upon which ice crystals can form, are present. The cleaning of dust particles carried by falling ice crystals (also known as scavenging) is therefore not the sole reason for the existence of supersaturation on Mars as previously proposed (19).

During the following season, the northern hemisphere exhibits saturation ratios greater than 10 after $L_{s} 330^{\circ}$, perhaps beginning around $L_{s} 315^{\circ}$, before the early phase of the $\mathrm{C}$ storm. The southern hemisphere exhibits a more prevalent supersaturation at the same time, in the form of a discrete supersaturated area stretching between 15 and $40 \mathrm{~km}$ altitude, with unsaturated air beneath it extending to the pole. The same feature reforms soon after the $\mathrm{C}$ storm, but slowly vanishes by the time of the spring equinox $\left(L_{s} 360^{\circ}\right)$.

In the 80-100 km part of the profiles shown in Figure 2D, a supersaturated layer seems to persist throughout the observing period. While the saturation state is generally less reliable in that altitude range (see Figure S1 (21)), this layer is distinctly observed at $L_{s} 190-200^{\circ}$ in the Northern hemisphere (Figure 1B), while water ice clouds are also observed. The existence of such high altitude supersaturation indicates efficient ascent of water to the upper atmosphere. Other regions of saturation are observed intermittently in both hemispheres in at 50-60 km. The temperature is erratic in this region due to atmospheric waves that generate higher temporal variability, causing fluctuations of more than $20 \mathrm{~K}$ in a few weeks. Because this region was previously filled with humid air during the GDS, cloud formation is enhanced. The clouds form in a similar configuration to the lower troposphere, as discussed above, characterized by the simultaneous presence of clouds and supersaturation. An alternative version of Figure 2, showing the averaged vmr and saturation ratio profiles binned into three narrow $L_{s}$ intervals, is shown in Figure S3 (21).

Our analysis constrains the mechanism that controls water propagation from the lower to the upper atmosphere. While dust controls upward propagation of water during the 2018 
GDS and the 2019 C storm, water vapor can effectively and persistently reach the upper atmosphere around perihelion in the southern hemisphere. This coincides with the seasonal intensification of the Hadley circulation that peaks up around $L_{s} 240^{\circ}$ and is active until $L_{s}$ $290^{\circ}$ (see also Figure S2, (21)).

Large portions of the atmosphere are in a state of supersaturation, complementing previous observations $(19,26)$. Unconstrained by saturation, the water vapor globally penetrates through the cloud level, regardless of the dust distribution, facilitating the loss of water to space. Because supersaturation is observed concomitantly with dust or ice particles, we conclude that condensation does not efficiently prevent water vapor from becoming super-saturated, even when seeds for condensation exist. We speculate this may be due to rapid drops in temperature and/or rises in water concentration, which occur faster than condensation can keep up with.

Our results also show that water access to high altitude is affected by the seasonal changes around perihelion. Although planetary-scale dust storms appear in this period, those irregular events have a lesser impact than seasonal change, which we suggest is the major atmospheric regulator for water. The seasonal recurrence and duration of the perihelion climate dominate the intermittent and short-lived effects of non-perihelion storms. Because perihelion coincides with the most intense period of the Hadley circulation, whose upwelling region is theoretically located in the southern tropical latitudes (27-30), and with the warmest period of the year, the perihelion season has likely governed the escape of water to space over geological timescales.

\section{References and Notes}

1. M. H. Carr, Water on Mars. Nature. 326, 30-35 (1987).

2. J. Lasue, N. Mangold, E. Hauber, S. Clifford, W. Feldman, O. Gasnault, C. Grima, S. 
Maurice, O. Mousis, Quantitative Assessments of the Martian Hydrosphere. Space Sci. Rev. 174, 155-212 (2013).

3. T. Owen, J. P. Maillard, C. de Bergh, B. L. Lutz, Deuterium on Mars - The abundance of HDO and the value of D/H. Science. 240, 1767-1770 (1988).

4. S. Aoki, H. Nakagawa, H. Sagawa, M. Giuranna, G. Sindoni, A. Aronica, Y. Kasaba, Seasonal variation of the $\mathrm{HDO} / \mathrm{H} 2 \mathrm{O}$ ratio in the atmosphere of Mars at the middle of northern spring and beginning of northern summer. Icarus. 260, 7-22 (2015).

5. G. L. Villanueva, M. J. Mumma, R. E. Novak, H. U. Kaufl, P. Hartogh, T. Encrenaz, A. Tokunaga, A. Khayat, M. D. Smith, Strong water isotopic anomalies in the martian atmosphere: Probing current and ancient reservoirs. Science. 348, 218-221 (2015).

6. M. B. McElroy, Mars: An Evolving Atmosphere. Science. 175, 443-445 (1972).

7. T. D. Parkinson, D. M. Hunten, Spectroscopy and Aeronomy of O2 on Mars. J. Atmospheric Sci. 29, 1380-1390 (1972).

8. V. A. Krasnopolsky, Mars' upper atmosphere and ionosphere at low, medium, and high solar activities: Implications for evolution of water: Mars’ upper atmosphere and ionosphere. J. Geophys. Res. Planets. 107, 11-1-11-11 (2002).

9. M. S. Chaffin, J.-Y. Chaufray, I. Stewart, F. Montmessin, N. M. Schneider, J.-L. Bertaux, Unexpected variability of Martian hydrogen escape: Geophys. Res. Lett. 41, 314-320 (2014).

10. J. T. Clarke, J.-L. Bertaux, J.-Y. Chaufray, G. R. Gladstone, E. Quemerais, J. K. Wilson, D. Bhattacharyya, A rapid decrease of the hydrogen corona of Mars: the Martian Hydrogen Corona. Geophys. Res. Lett. 41, 8013-8020 (2014).

11. M. S. Chaffin, J. Deighan, N. M. Schneider, A. I. F. Stewart, Elevated atmospheric escape of atomic hydrogen from Mars induced by high-altitude water. Nat. Geosci. 10, 174-178 (2017). 
12. N. G. Heavens, A. Kleinböhl, M. S. Chaffin, J. S. Halekas, D. M. Kass, P. O. Hayne, D. J. McCleese, S. Piqueux, J. H. Shirley, J. T. Schofield, Hydrogen escape from Mars enhanced by deep convection in dust storms. Nat. Astron. 2, 126-132 (2018).

13. V. A. Krasnopolsky, Photochemistry of water in the martian thermosphere and its effect on hydrogen escape. Icarus. 321, 62-70 (2019).

14. D. V. Michelangeli, O. B. Toon, R. M. Haberle, J. B. Pollack, Numerical simulations of the formation and Evolution of water ice clouds in the Martian atmosphere. Icarus. 100, 261-285 (1993).

15. R. T. Clancy, A. W. Grossman, M. J. Wolff, P. B. James, D. J. Rudy, Y. N. Billawala, B. J. Sandor, S. W. Lee, D. O. Muhleman, Water vapor saturation at low latitudes around aphelion: A key to Mars climate? Icarus. 122, 36-62 (1996).

16. T. Navarro, J.-B. Madeleine, F. Forget, A. Spiga, E. Millour, F. Montmessin, A. Määttänen, Global climate modeling of the Martian water cycle with improved microphysics and radiatively active water ice clouds. J. Geophys. Res. Planets. 119, 1479-1495 (2014).

17. O. Korablev, F. Montmessin, A. Trokhimovskiy, A. A. Fedorova, A. V. Shakun, A. V. Grigoriev, B. E. Moshkin, N. I. Ignatiev, F. Forget, F. Lefèvre, K. Anufreychik, I. Dzuban, Y. S. Ivanov, Y. K. Kalinnikov, T. O. Kozlova, A. Kungurov, V. Makarov, F. Martynovich, I. Maslov, D. Merzlyakov, P. P. Moiseev, Y. Nikolskiy, A. Patrakeev, D. Patsaev, A. Santos-Skripko, O. Sazonov, N. Semena, A. Semenov, V. Shashkin, A. Sidorov, A. V. Stepanov, I. Stupin, D. Timonin, A. Y. Titov, A. Viktorov, A. Zharkov, F. Altieri, G. Arnold, D. A. Belyaev, J. L. Bertaux, D. S. Betsis, N. Duxbury, T. Encrenaz, T. Fouchet, J.-C. Gérard, D. Grassi, S. Guerlet, P. Hartogh, Y. Kasaba, I. Khatuntsev, V. A. Krasnopolsky, R. O. Kuzmin, E. Lellouch, M. A. Lopez-Valverde, M. Luginin, A. Määttänen, E. Marcq, J. Martin Torres, A. S. Medvedev, E. Millour, K. S. Olsen, M. R. 
Patel, C. Quantin-Nataf, A. V. Rodin, V. I. Shematovich, I. Thomas, N. Thomas, L. Vazquez, M. Vincendon, V. Wilquet, C. F. Wilson, L. V. Zasova, L. M. Zelenyi, M. P. Zorzano, The Atmospheric Chemistry Suite (ACS) of Three Spectrometers for the ExoMars 2016 Trace Gas Orbiter. Space Sci. Rev. 214 (2018), doi:10.1007/s11214-0170437-6.

18. Fedorova, O. I. Korablev, J.-L. Bertaux, A. V. Rodin, F. Montmessin, D. A. Belyaev, A. Reberac, Solar infrared occultation observations by SPICAM experiment on MarsExpress: Simultaneous measurements of the vertical distributions of $\mathrm{H} 2 \mathrm{O}, \mathrm{CO} 2$ and aerosol. Icarus. 200, 96-117 (2009).

19. L. Maltagliati, F. Montmessin, A. Fedorova, O. Korablev, F. Forget, J.-L. Bertaux, Evidence of Water Vapor in Excess of Saturation in the Atmosphere of Mars. Science. 333, 1868-1871 (2011).

20. Fedorova, J.-L. Bertaux, D. Betsis, F. Montmessin, O. Korablev, L. Maltagliati, J. Clarke, Water vapor in the middle atmosphere of Mars during the 2007 global dust storm. Icarus. 300, 440-457 (2018).

21. Materials and Methods are available as supplementary material.

22. The solar longitude Ls is the Mars-Sun angle in degrees, measured from the northern hemisphere spring equinox, where $\mathrm{Ls}=0^{\circ}$. Ls $=270^{\circ}$ marks the northern winter solstice. Perihelion, where Mars orbits the closest to the Sun, occurs at Ls $=251^{\circ}$.

23. S. D. Guzewich, M. Lemmon, C. L. Smith, G. Martínez, Á. Vicente-Retortillo, C. E. Newman, M. Baker, C. Campbell, B. Cooper, J. Gómez-Elvira, A. -M. Harri, D. Hassler, F. J. Martin-Torres, T. McConnochie, J. E. Moores, H. Kahanpää, A. Khayat, M. I. Richardson, M. D. Smith, R. Sullivan, M. Torre Juarez, A. R. Vasavada, D. Viúdez-Moreiras, C. Zeitlin, M. Zorzano Mier, Mars Science Laboratory Observations of the 2018/Mars Year 34 Global Dust Storm. Geophys. Res. Lett. 46, 71-79 (2019). 
24. D.M. Kass, A. Kleinböhl, D.J. McCleese, J. T. Schofield, M.D. Smith, Interannual similarity in the Martian atmosphere during the dust storm season, Geophys. Res. Lett. 43, 6111- 6118 (2016).

25. P. Wolkenberg, M. Giuranna, D. Grassi, A. Aronica, S. Aoki, D. Scaccabarozzi, B. Saggin, Characterization of dust activity on Mars from MY27 to MY32 by PFS-MEX observations. Icarus. 310, 32-47 (2018).

26. R. Todd Clancy, M. D. Smith, F. Lefèvre, T. H. McConnochie, B. J. Sandor, M. J. Wolff, S. W. Lee, S. L. Murchie, A. D. Toigo, H. Nair, T. Navarro, Vertical profiles of Mars $1.27 \mu \mathrm{m}$ O 2 dayglow from MRO CRISM limb spectra: Seasonal/global behaviors, comparisons to LMD-GCM simulations, and a global definition for Mars water vapor profiles. Icarus. 293, 132-156 (2017).

27. F. Forget, F. Hourdin, R. Fournier, C. Hourdin, O. Talagrand, M. Collins, S. R. Lewis, P. L. Read, J. Huot, Improved general circulation models of the Martian atmosphere from the surface to above $80 \mathrm{~km}$. J. Geophys. Res. Planets. 104, 24155-24176 (1999).

28. M. I. Richardson, R. J. Wilson, A topographically forced asymmetry in the martian circulation and climate. Nature. 416, 298-301 (2002).

29. F. Montmessin, F. Forget, P. Rannou, M. Cabane, R. M. Haberle, Origin and role of water ice clouds in the Martian water cycle as inferred from a general circulation model. $J$. Geophys. Res. Planets. 109, 10004 (2004).

30. D. S. Shaposhnikov, A. S. Medvedev, A. V. Rodin, P. Hartogh, Seasonal Water "Pump” in the Atmosphere of Mars: Vertical Transport to the Thermosphere. Geophys. Res. Lett. 46, 4161-4169 (2019).

31. A.C. Vandaele, J.J. Lopez-Moreno, M.R. Patel et al., NOMAD, an Integrated Suite of Three Spectrometers for the ExoMars Trace Gas Mission: Technical Description, Science Objectives and Expected Performance, Space Sci Rev 214, 80 (2018), 
https://doi.org/10.1007/s11214-018-0517-2

32. I.E. Gordon et al., The HITRAN2016 molecular spectroscopic database. Journal of Quantitative Spectroscopy \& Radiative Transfer 203, 3-69 (2017).

33. L. R. Brown, C. M. Humphrey, R. R. Gamache, $\mathrm{CO}_{2}$-broadened water in the pure rotation and $v_{2}$ fundamental regions. Journal of Molecular Spectroscopy 246, 1-21 (2007).

34. C. D. Rodgers, Inverse methods for atmospheric sounding: Theory and practice. (Vol.2 in Series on atmospheric, oceanic and planetary physics, F.W.Taylor, Eds., World Scientific Publishing Co.Pte.Ltd., Singapure, 2000).

35. K. Levenberg, A method for the solution of certain non-linear problems in least squares. Quarterly Journal of Applied Mathematics, 164-168 (1944).

36. D. Marquardt, An Algorithm for Least-Squares Estimation of Nonlinear Parameters. Journal of the Society for Industrial and Applied Mathematics 11, 431-441 (1963).

37. Mars Climate Database v5.3: http://www-mars.lmd.jussieu.fr/mars/access.html

38. S. Ceccherini, Analytical determination of the regularization parameter in the retrieval of atmospheric vertical profiles, Opt. Lett. 30, 2554-2556 (2005)

39. S. Ceccherini, C. Belotti, B. Carli, P. Raspollini, and M. Ridolfi.: Technical Note: Regularization performances with the error consistency method in the case of retrieved atmospheric profiles, Atmos. Chem. Phys. 7, 1435-1440, https://doi.org/10.5194/acp-71435-2007 (2007).

40. Kleinböhl et al., Mars Climate Sounder limb profile retrieval of atmospheric temperature, pressure, and dust and water ice opacity. Journal of Geophysical Research (Planets) 114, 10006 (2009).

41. P. Irwin, N. Teanby, R. de Kok, L. Fletcher, C. Howett, C. Tsang, C. Wilson, S. Calcutt, C. Nixon, and P. Parrish, The NEMESIS planetary atmosphere radiative transfer and retrieval tool. Journal of Quantitative Spectroscopy and Radiative Transfer, 109(6):1136- 
1150. https://doi.org/10.1016/j.jqsrt.2007.11.006 (2008).

42. J. Alday et al., Oxygen isotopic ratios in Martian water vapour observed by ACS MIR on board ExoMars Trace Gas Orbiter. Astronomy and Astrophysics, submitted (2019).

43. McCleese D.J. and Schofield J.T., MRO MARS CLIMATE SOUNDER LEVEL 5 DDR V1.0, NASA Planetary Data System, MRO-M-MCS-5-DDR-V1.0, 2006. (https://pds.nasa.gov/datasearch/subscription-service/SS-20190601.shtml)

44. R. J. List, Smithsonian Institution, Smithsonian meteorological tables. (Smithsonian Institution Press, Washington, 1984).

45. J. A. Goff, and S. Gratch, Low-pressure properties of water from -160 to $212 \mathrm{~F}$, Transactions of the American Society of Heating and Ventilating Engineers, 25-164, New York (1946).

46. M. L. Clapp, D. R. Worsnop, and R. E. Miller, Frequency-dependent optical constants of water ice obtained directly from aerosol extinction spectra. The Journal of Physical Chemistry, 99(17), 6317-6326. http://doi.org/10.1021/j100017a010 (1995).

47. S. G. Warren and R.E. Brandt, Optical constants of ice from the ultraviolet to the microwave: A revised compilation. Journal of Geophysical Research, 113(D14), D14220. http://doi.org/10.1029/2007JD009744 (2008).

48. M. J. Wolff, M. D. Smith, R. T. Clancy, R. Arvidson, M. Kahre, F. Seelos, S. Murchie, H. Savijärvi, Wavelength dependence of dust aerosol single scattering albedo as observed by the Compact Reconnaissance Imaging Spectrometer. Journal of Geophysical Research, 114(6), E00D04. http://doi.org/10.1029/2009JE003350 (2009).

49. J. E. Hansen and L. D. Travis, Light scattering in planetary atmospheres. Space Science Reviews 16, 527-610. https://doi.org/10.1007/BF00168069 (1974). 
Acknowledgments: ExoMars is a joint space mission by the European Space Agency (ESA) and Roscosmos. The ACS experiment is led by the Space Research Institute (IKI) in Moscow, assisted by LATMOS in France. We thank the numerous people responsible for designing, building, testing, launching, communicating with and operating the spacecraft and science instruments of TGO.

Funding: The project acknowledges funding by Roscosmos and Centre National d'Etudes Spatiales (CNES). The science operations of ACS are funded by Roscosmos and ESA. Authors affiliated with IKI acknowledge funding from the Russian government under grant number 14.W03.31.0017 and contract number 0120.0602993 (0028-2014-0004). Authors affiliated with the University of Oxford acknowledge funding from the UK Space Agency under grants ST/R001502/1 and ST/P001572/1. Authors affiliated with LATMOS acknowledge funding from CNES and Centre National de la Recherche Scientifique (CNRS). K.O. acknowledge the Natural Sciences and Engineering Research Council of Canada Grant Number: PDF - 516895 - 2018.

Author contributions: A.F., F.M., and O.K. conceived the study, collected inputs of other co-authors and wrote the paper. The ACS observations and raw dataset were prepared by A.T., A.G., A.S., A.P. and N.K.. A.F. calibrated the NIR ACS data and analyzed the profiles (assisted by A.T. and J.-L.B.). N.I. provided TIRVIM calibrated data. A.T., K.S.O. and L.B provided MIR calibrated data. D.B. provided the ACS MIR aerosol extinction profiles. M.L. provided retrieval of aerosols properties based on TIRVIM, NIR and MIR dataset. J.A. and P.G.J.I. provided the MIR temperatures for validations. S.K. validated the MCS dataset. F.M., F.L., F.F., E.M., A.M. and C.W. provided expertise on the chemistry, circulation and microphysics (assisted by J.L.B.). All authors contributed to the preparation of the manuscript.

Competing interests: The authors declare no competing interests. 
Data and materials availability: ACS data are available from ESA Planetary Science Archive (PSA) Level 2 https://archives.esac.esa.int/psa/\#!Table\%20View/ACS=instrument (21). The temperature, $\mathrm{H}_{2} \mathrm{O}$ and aerosol extinction profiles retrieved from the ACS measurements and used in this article are available on the IKI FTP server ftp://193.232.13.174/ACS_NIR_Results/ .

\section{Supplementary Materials}

Materials and methods

Figures S1 to S14

Table S1 to S3

References (31-49) 
Fig. 1: Example ACS spectra and retrieved profiles. (A) ACS NIR spectra measured during orbit $2580\left(\mathrm{Ls}=197.8^{\circ}, 47.27^{\circ} \mathrm{N}, 85.2^{\circ} \mathrm{W}\right.$, local time $\left.17: 27\right)$ at three example altitudes, labelled in each panel. Synthetic models (blue curves) fitted to the data (red dots) account for the water content, $\mathrm{CO}_{2}$ number density, and atmospheric temperature. The residuals are shown with gray lines. (B) Corresponding retrieved profiles of the $\mathrm{H}_{2} \mathrm{O}$ mixing ratio, temperature, and saturation ratio derived from them. The profiles of the mass of aerosol particles (ice-blue circles; dust - red dots) per $\mathrm{cm}^{3}$ obtained during the same orbit from ACS TIRVIM data are also shown. All altitudes are above areoid (equipotential surface for Mars, the analog of geoid for the Earth). See (21) for details of the model fitting, retrieval procedures. The error bars or shaded regions, corresponding to $1 \sigma$ confidence, are also explained in (21).

Fig. 2: Derived atmospheric properties during the dusty season of MY34. Each panel shows the data value in color, plotted as functions of Ls and altitude, with the Northern hemisphere on the left and the Southern hemisphere on the right. (A) Distribution of the ACS-NIR solar occultation observations, showing morning (red) and evening (blue) events.

(B) Atmospheric temperature. (C) Water vapor mixing ratio. (D) Saturation ratio of water vapor; the grey regions correspond to an undersaturated state (i.e. saturation ratio $<1)$. (E) Water ice (blue) and dust (brown) aerosol extinctions. 
A $7230 \quad 7250 \quad 7270 \quad 7290$
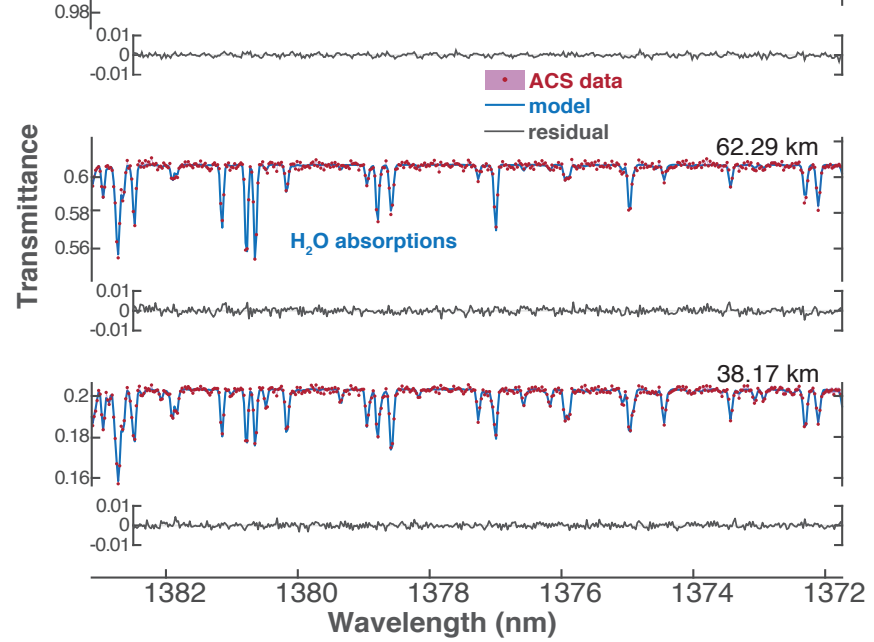

B

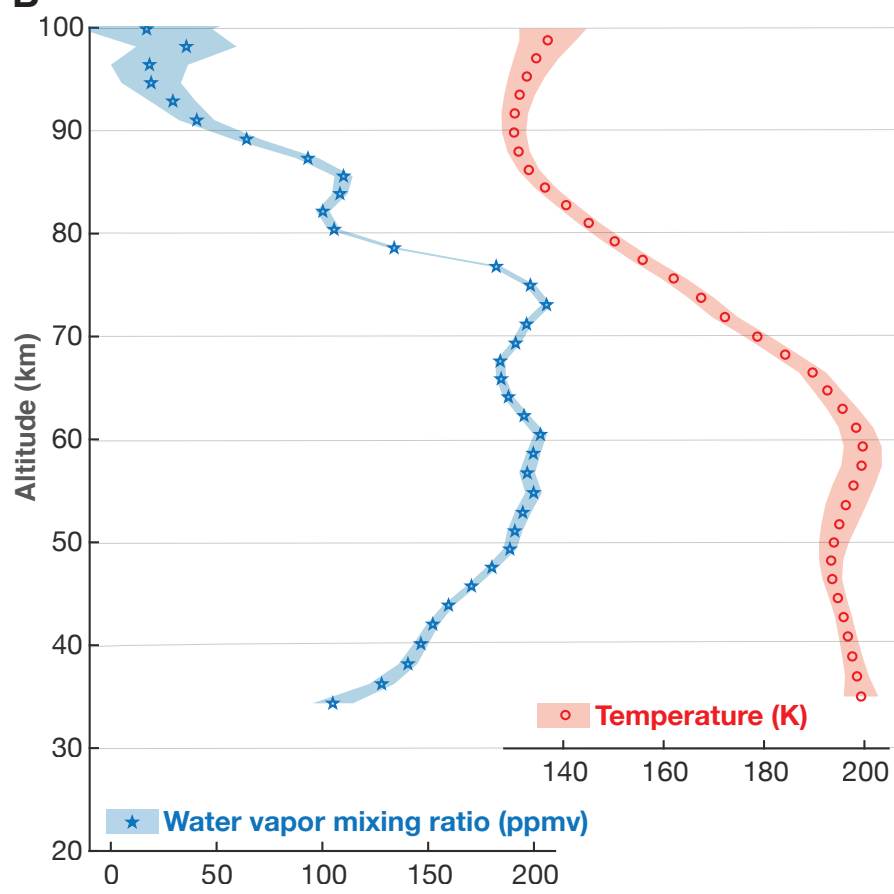

Wavenumber $\left(\mathrm{cm}^{-1}\right)$
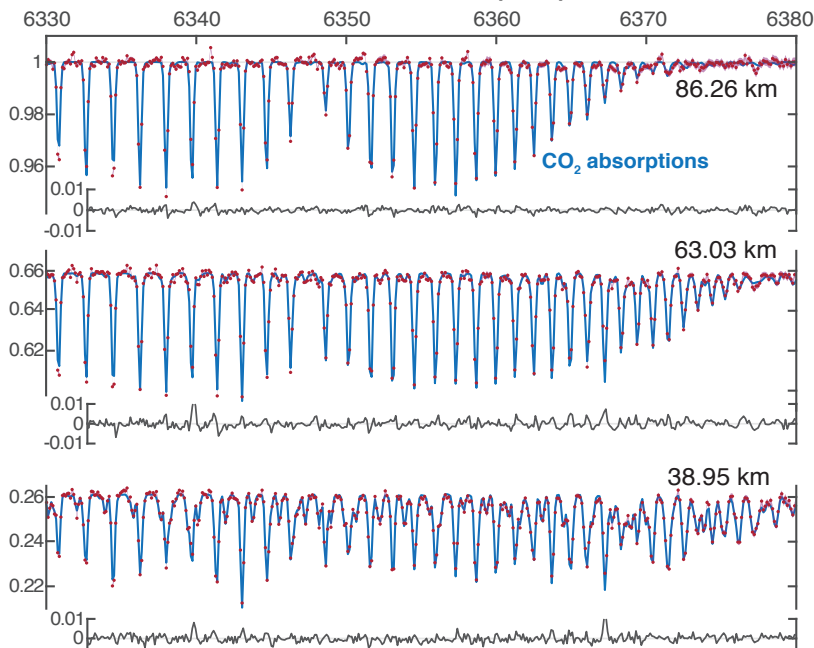

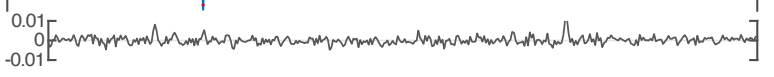

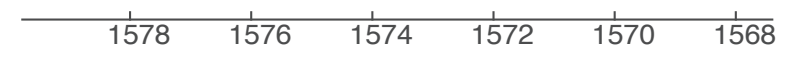

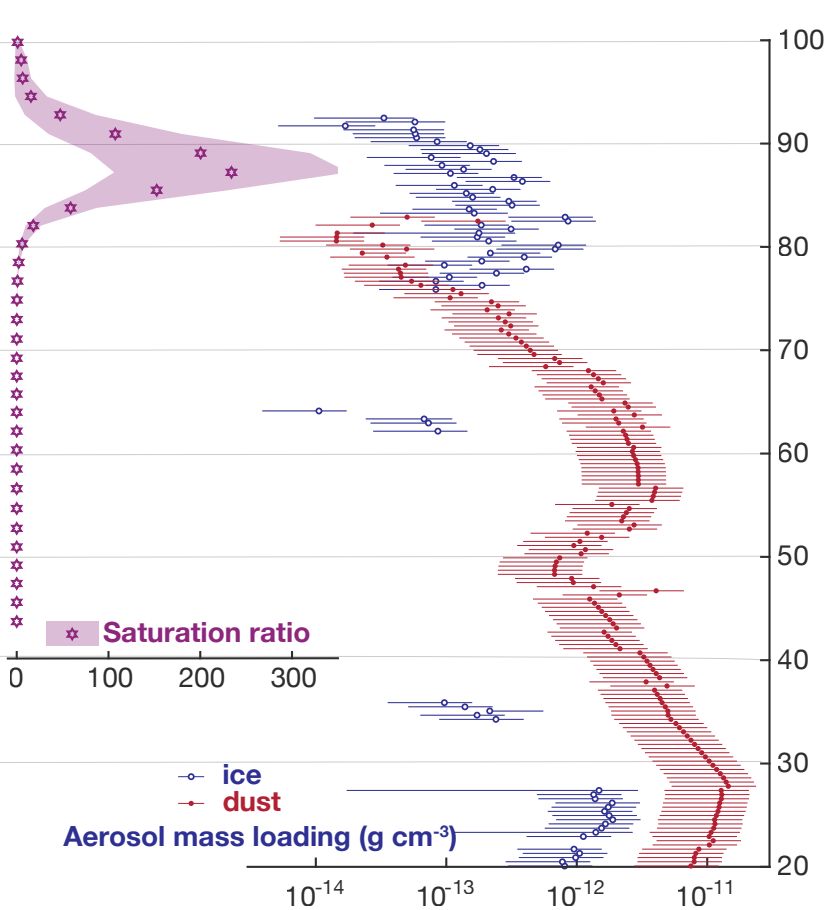


Northern hemisphere

A

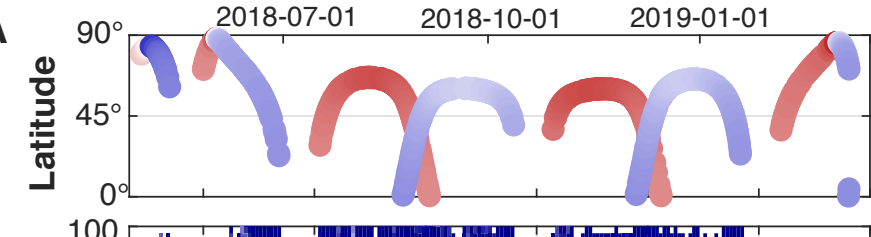

B

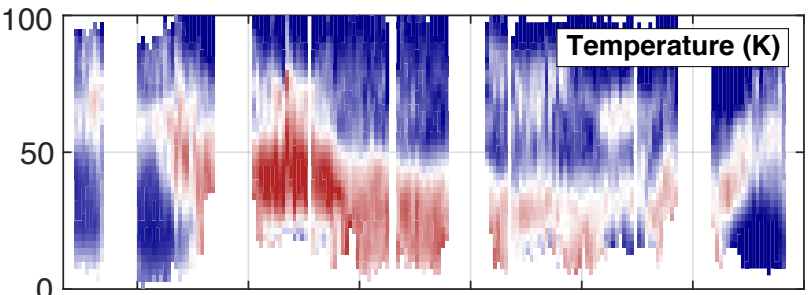

C

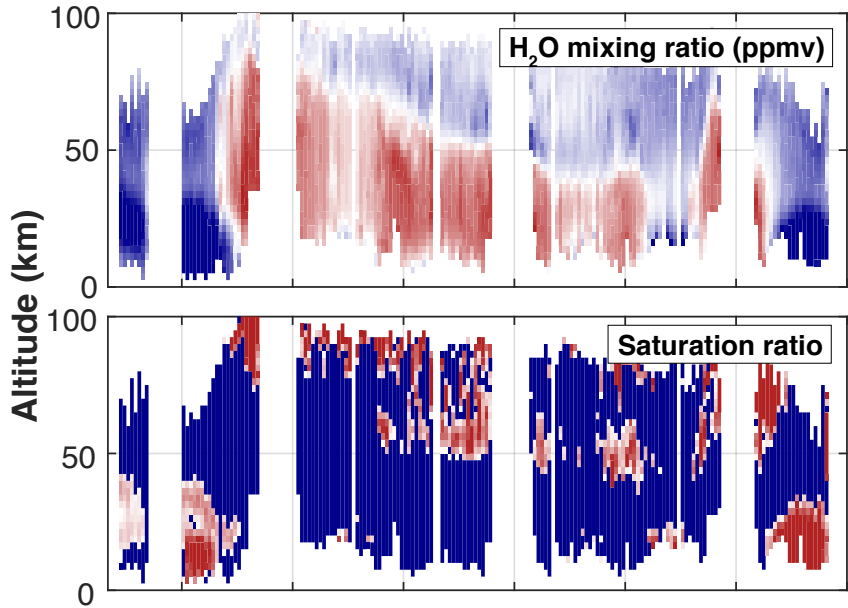

E

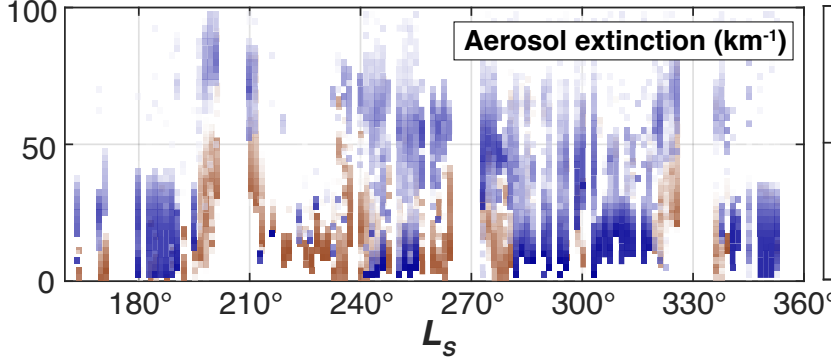

Southern hemisphere
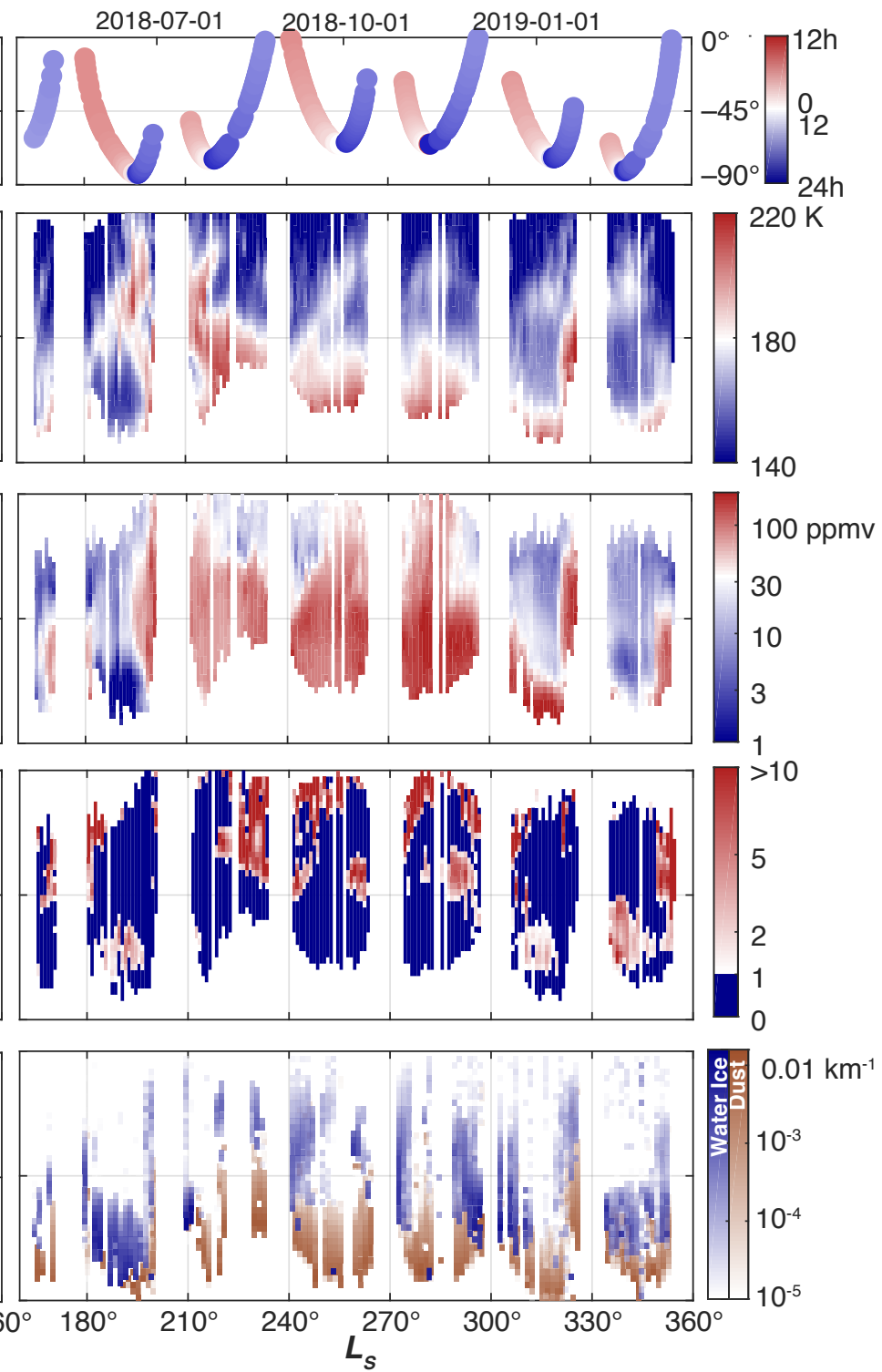


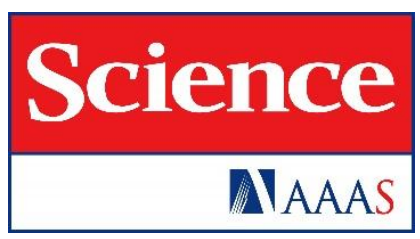

\section{Supplementary Materials for}

Stormy water on Mars: the distribution and saturation of atmospheric water during the dusty season

Anna A. Fedorova, Franck Montmessin, Oleg Korablev, Mikhail Luginin, Alexander Trokhimovskiy, Denis A. Belyaev, Nikolay I. Ignatiev, Franck Lefèvre, Juan Alday,

Patrick G.J. Irwin, Kevin S. Olsen, Jean-Loup Bertaux, Ehouarn Millour, Anni

Määttänen, Alexey Shakun, Alexey V. Grigoriev, Andrey Patrakeev, Svyatoslav Korsa, Nikita Kokonkov, Lucio Baggio, Francois Forget, Colin F. Wilson.

correspondence to: fedorova@iki.rssi.ru

This PDF file includes:

Materials and Methods

Figs. S1 to S14

Tables S1 to S3 


\section{Materials and Methods}

The ACS instrument and dataset

The ACS instrument (17) consists of three infrared spectrometers covering a broad spectral range (0.7 to $17 \mu \mathrm{m})$. ACS NIR couples an acousto-optical tunable filter (AOTF) used as a monochromator to isolate the wavelength bandpass sampled by an echelle spectrometer operated at high diffraction orders. ACS NIR covers a spectral range of 0.7$1.7 \mu \mathrm{m}$ in diffraction orders 101 through 49. During an occultation, ACS NIR measures 10 preselected diffraction orders in two seconds, including the absorption bands of $\mathrm{H}_{2} \mathrm{O}$ at $1.13,1.38$, and $1.40 \mu \mathrm{m}$, and $\mathrm{CO}_{2}$ at $1.27,1.43,1.54$, and $1.57 \mu \mathrm{m}$. The instantaneous field-of-view (FOV) in the direction perpendicular to the limb is small $\left(\sim 0.02^{\circ}\right)$, and limited by the slit width. This corresponds to an instantaneous vertical resolution of 500$600 \mathrm{~m}$ at the tangent heights we observed. The time to measure one diffraction order is 0.2 seconds, that includes the integration of signal with AOTF on and with AOTF off, readout time and accumulation of 8 individual exposures; the repetition rate is $2 \mathrm{~s}$. The effective vertical resolution at the limb depends on the beta-angle (the angle between the orbit plane and the direction to the Sun) and for each diffraction order is generally better than $1 \mathrm{~km}$. Horizontal resolution (along the slit) is determined by number (from 6 to 20) of detector lines being stacked and amounts to $1.5-5 \mathrm{~km}$. We have used the diffraction order $56\left(7220-7300 \mathrm{~cm}^{-1}\right)$ to retrieve water vapor and the diffraction order 49 (6318$6387 \mathrm{~cm}^{-1}$ ) to retrieve the $\mathrm{CO}_{2}$ density and temperature of the atmosphere. The continuum absorption in orders 78 and 90 (around 10,010 and 11,565 $\mathrm{cm}^{-1}$, respectively) was used to assess the aerosol extinction.

The thermal infrared (TIRVIM) channel is a Fourier-transform spectrometer with a cryogenic detector and the solar occultation capability. In occultation, TIRVIM operates mostly in "climatology" mode, covering the full spectral range of 1.7-17 $\mu \mathrm{m}$ every $0.4 \mathrm{~s}$, with a spectral resolution of $\leq 1 \mathrm{~cm}^{-1}$. The TIRVIM's circular FOV of $2.5^{\circ}$ diameter is larger than the solar disk. The vertical resolution, determined by the angular diameter of the Sun, is close to one atmospheric scale height $(\sim 10 \mathrm{~km})$. We used the continuum absorption data in the range of $1700-5000 \mathrm{~cm}^{-1}(2-6 \mu \mathrm{m})$, where the signal-to-noise ratio is higher, and the solar signal dominates over the thermal emission of the planet.

The mid-infrared (MIR) channel is a crossed dispersion echelle spectrometer dedicated to solar occultation measurements in the 2.3-4.5 $\mu \mathrm{m}$ range with spectral resolving power of 30000. For each acquired frame, MIR measures up to 20 adjacent diffraction orders, covering an instantaneous spectral range of 0.15-0.3 $\mu \mathrm{m}$. To achieve the full spectral coverage a secondary dispersion grating can be rotated to one of 11 positions. During a given occultation MIR is operated in one pre-chosen secondary grating position. We used the integrated signal in orders 192 and 175 within the secondary grating position 12 to characterize the continuum absorption of dust and $\mathrm{H}_{2} \mathrm{O}$ ice in its 3- $\mu \mathrm{m}$ absorption band. The FOV of MIR is small; its vertical resolution is defined by the integration time and amounts to $2.5 \mathrm{~km}$. The vertical sampling of MIR was increased twofold by using the detector lines corresponding to the lower and upper parts of the slit.

TGO observes each occultation with one or both of two instruments: ACS and NOMAD (Nadir and Occultation for Mars Discovery) (31). Because of a subtle misalignment of the ACS and NOMAD lines-of-sight (LOS), two occultation observing 
modes are implemented: ACS MIR-driven and NOMAD-driven. ACS MIR can be operated only with the ACS pointing, while NIR and TIRVIM can use both kinds of pointing. We used all the NIR occultation profiles obtained both with the ACS and NOMAD pointings (the latter constituting $~ 10 \%$ of profiles). The TIRVIM occultation dataset, generally well-sampled in time, has gaps at some periods $\left(L_{s} 212-240^{\circ}, 255-\right.$ $265^{\circ}$ ), and becomes thinner later, because of instrument problems, and due to a new operation scheme, implemented from $L_{s} 275^{\circ}$ onwards and intended to spare the operational time of the detector's cryogenic cooler. To fill the gaps we have combined the dataset with the aerosol extinction data from the ACS MIR channel, characterizing the water ice band near $3 \mu \mathrm{m}$. The short-wavelength half of the $\mathrm{H}_{2} \mathrm{O}$ ice band is also measured in MIR position 13, but this position was only used at the beginning of the mission and later decided to be of a lower priority than other positions; the measurements were discontinued after $L_{s} 280^{\circ}$. For consistency, we therefore used MIR data from position 12 only. The full ACS dataset used in this study is depicted in Figure S4; a summary is given in Table S1.

ACS low-level data are available from ESA Planetary Science Archive (PSA) https://archives.esac.esa.int/psa/\#!Table\%20View/ACS=instrument, where the intensity spectra measured by ACS for each of the abovementioned orbits can be identified and retrieved. The data is following Planetary Data System Version 4 (PDS4) standards. The files are composed of a .xml (label) file which references a raw packets files and lists appropriate attributes.

\section{Temperature and water vapor retrieval}

The procedure to obtain the water mixing ratio profiles from the occultation data is described in refs. (18-20). The $\mathrm{CO}_{2}$ and $\mathrm{H}_{2} \mathrm{O}$ abundances from respectively the 1.57 and $1.38 \mu \mathrm{m}$ spectral regions are measured in parallel with a slight altitude shift due to sequential measurements of the diffraction orders, which is accounted for using interpolation.

On the calibrated NIR transmission data, first, the wavelength drift is corrected using positions of gaseous absorption lines. A forward model of transmission is computed using a look-up-table of absorption cross-sections (as a function of pressure and temperature) for a corresponding number of atmospheric layers (40 to 130 depending on orbit), using the spectral line parameters from the HITRAN 2016 database (32) with a correction coefficient of 1.7 for the $\mathrm{H}_{2} \mathrm{O}$ broadening in $\mathrm{CO}_{2}$-dominated atmosphere (33) and self-broadening in the case of $\mathrm{CO}_{2}$.

On the first step, the model fitting is performed on the data of the diffraction order 49 (6318-6387 $\mathrm{cm}^{-1}$ ), which covers a $\mathrm{CO}_{2}$ absorption band, including multiple temperature-sensitive lines with different ground-state energy ( $\left.E^{\prime \prime}\right)$ (Fig. S5A). NIR resolves the rotational structure of the $\mathrm{CO}_{2}$ band, enabling retrieval of both temperature and pressure profiles. An additional free parameter is the $\mathrm{CO}$ local density; this produces a weak absorption being present within the spectral range, superimposed over the dominating $\mathrm{CO}_{2}$ structure. The retrieval algorithm returns these variables at all altitudes at the same time, i.e. the vertical profiles. We use a Levenberg-Marquardt iterative scheme (34-36), minimizing the square of $Y-f(X)$, where the data vector $Y$ contains all measured spectra at all altitudes and is sized as $N \times M$, where $N$ is the number of altitudes and $M$ is the number of spectral points (640). The state vector $X$, with size $3 \times N$, contains 
values of temperature, pressure and the volume mixing ratio $(\mathrm{vmr})$ of $\mathrm{CO}\left(f_{\mathrm{CO}}\right)$ at all altitudes. As an initial assumption for the temperature $(T)$ and pressure $(P)$, we used the Martian Climate Database MCD 5.3 profiles (37). The model includes several dust scenarios. The "climatology" scenario is developed from the available observations of dust from multiple Martian years (MY 24, 26, 27, 29, 30, 31) without global planetencircling dust storms. The "cold," or low-dust scenario assumes the lowest dust opacity at a given location and Ls out of the same six relatively quiet Mars years, further divided by two. The "warm" scenario sets the dust opacity to the maximum observed the six years. The dust storm scenario ("dust”) imitates Mars during the extreme global dust storms (observed in MY25 and MY28). Our retrieval is based on the "climatology" scenario. The initial mixing ratio of $\mathrm{CO}$ was assumed to be $1000 \mathrm{ppm}$ at all altitudes. The model does not fit the $\mathrm{CO}_{2}$ volume mixing ratio, instead we assume its vmr is constant up to $100 \mathrm{~km}$. However, in polar regions, the $\mathrm{CO}_{2} \mathrm{vmr}$ is predicted by the MCD to decrease above $80 \mathrm{~km}$, which can impact the retrieval.

The gradient of radiance with the state vector elements for all spectral points and altitudes is given by the Jacobian matrix of partial derivatives. This was calculated for all components of $X$, and therefore consists of three parts, $\partial \operatorname{Tr} / \partial T, \partial \operatorname{Tr} / \partial P$ and $\partial \operatorname{Tr} / \partial f_{\mathrm{CO}}$ where $\operatorname{Tr}$ is the transmittance. It has a size of $(3 N \times M N)$. The corresponding Jacobian matrices take into account the dependence of the absorption cross-sections on $T$ and $P$. Once the solution, the vector $X$, is obtained, Tikhonov regularization is applied, as is common for vertical inversions to smooth the profile and minimize the uncertainties (3839). The obtained smoothed profiles serve as an initial assumption for the following iteration with Levenberg-Marquardt. The algorithm converges within 3-6 iterations independently of initial assumptions, as shown in Fig. S6. The Jacobian matrix is sensitive to the temperature profile due to the pronounced $\mathrm{CO}_{2}$ rotational structure in our spectral range. The uncertainty in the retrieved profiles is given by the covariance matrix of the solution errors.

The retrieval of the vertical profiles of pressure with the rotational structure of the $\mathrm{CO}_{2}$ band generates sometimes unrealistic results. In $\sim 30 \%$ of profiles of the analyzed dataset, we observed a bend, a drop of the pressure curve at some altitudes, which contradicts with the hydrostatic law. Therefore, in the second step, we used the assumption of hydrostatic equilibrium, constraining simultaneous retrieval of temperature and pressure. We determine a pressure $P_{0}$ at an altitude level $z_{0}$ where the uncertainties are minimal (the highest data quality), and then compute the pressure profile from the hydrostatic equilibrium, integrating the retrieved $T$ profile above and below the $P_{0}$ level:

$$
P(h)=P_{o} \exp \left(-\int_{z 0}^{h} \frac{g(z) M(z)}{T(z) k} d z\right) \text {, }
$$

where $g(z)$ is the acceleration due to gravity, $M(z)$ is the molecular weight (taken from MCD), $k$ is the Boltzmann constant, $z$ is the altitude, $h$ is the altitude level of pressure $P$. Once the pressure is calculated, we repeat the temperature retrieval (as described for Step 1, with only two free parameters, the pressure profile being fixed), and perform several iterations until the profiles of pressure and temperature converge. The comparison of the temperature profiles obtained on the first step (rotational) and the second step (hydrostatic) is shown in Fig. S7.

In the third step, the $\mathrm{H}_{2} \mathrm{O}$ number density and vmr are retrieved applying a similar retrieval procedure to the spectra in the diffraction order 56 (7217-7302 $\mathrm{cm}^{-1}$ ) encompassing the 1.38- $\mu \mathrm{m}$ water vapor band (see Figure 1). Only one free parameter 
vector is retrieved (the $\mathrm{H}_{2} \mathrm{O}$ vmr). The pressure and temperature profiles are prescribed, using the results of Step 2.

This self-consistent retrieval of the $\mathrm{H}_{2} \mathrm{O}$ profile along with that of $\mathrm{CO}_{2}$ and the temperature profile contracts with the MCD, where the profiles of the atmosphere at terminator are poorly constrained. Without this self-consistency, the vmr may be wrongly estimated and the strong dependence of water absorption on temperature may contribute to an error in estimating abundances (see Fig. S5B). Retrieved profiles of $\mathrm{H}_{2} \mathrm{O}$ vmr and temperature differ from the MCD predictions (37) as demonstrated in Fig. S6 and S8.

At the first and third steps of the procedure, the uncertainty on the retrieved quantities is given by the covariance matrix of the solution. In the second step, the uncertainty of $P o$ is also taken into account. In the third step, we account for the Jacobian errors due to the retrieved $T$ and $P$. For water vapor, the retrieval accuracy follows the theoretical prediction (17, their figure 30). It sharply depends on the aerosol loading, and, for a clear atmosphere (with an optical depth $\leq 0.2$ ), remains better than 1 ppmv at 10-50 $\mathrm{km}$.

\section{Validation of temperature profiles}

The NIR terminator temperature profiles were verified against similarly retrieved profiles from the MIR channel, and the collocated Mars Climate Sounder (MCS) results onboard Mars Reconnaissance Orbiter (MRO) (40). The MIR profiles were obtained using a weak $\mathrm{CO}_{2}$ band within secondary grating position 5 (3750-3950 cm-1), superimposed over a strong $\mathrm{H}_{2} \mathrm{O}$ band. The retrieval using Nemesis code (41) involves multiple variables including the temperature (42). The $\mathrm{H}_{2} \mathrm{O}$ profiles retrieved from NIR and MIR are consistent between the retrievals from different spectral ranges using two independent inversion schemes (Fig. S9).

The MCS/MRO profiles are selected from the PDS database (43), applying criteria on collocation (within $\pm 1^{\circ}$ latitude and longitude), local time ( 2 hours), and season (within $0.5^{\circ}$ of $L_{s}$ ). MRO observes the limb of Mars at 3 am and $3 \mathrm{pm}$, far from the terminator, except for the high-latitude areas. This makes the comparison of ACS and MCS temperatures difficult, though they are often similar (Figure S10). Due to the wave activity in the Martian atmosphere, the local time variation can be high. Figure S10C shows selected MCS profiles demonstrate a small shift with respect to the NIR profile, progressing with the local time. The comparison with MCS demonstrates that there is no substantial bias between the two, otherwise very different, limb datasets.

\section{$\underline{\text { Saturation ratio }}$}

The water vapor saturation pressure depends solely on the temperature in the approximation of the Clausius-Clapeyron relation (44), which is valid at first order in planetary atmospheres. For the computation of the water saturation state of the Martian atmosphere with respect to ice, we use a semi-empirical form of the Clausius-Clapeyron law, the Goff-Gratch equation $(44,45)$, as used by the World Meteorological Organization:

$$
\log P_{\text {sat }}=2.07023-0.00320991 T-2484.896 T^{-1}+3.56654 \log T \text {, }
$$
Kelvin.

where $P_{\text {sat }}$ is the saturation vapor pressure in mbar and $T$ is the temperature in 
The saturation ratio $S$ is defined as $S=P_{H 2 O} / P_{\text {sat }}$. The uncertainties of $S$ are evaluated as the total differential of $S$, using the uncertainties of the retrieved temperature, pressure and the water vmr. In Figures 2 all data with $\mathrm{H}_{2} \mathrm{O}$ uncertainties exceeding $100 \%$ or temperature error exceeding $20 \mathrm{~K}$ were excluded.

\section{Aerosol loading estimation}

Aerosol concentrations were retrieved from TIRVIM solar occultation data with addition, if available, of NIR and MIR channels data. The retrieval methodology (18) allows to disentangle the condensate clouds from the mineral dust and to constrain an intermediate case with a unimodal or bimodal distribution of the particle size. Extinction profiles were obtained at 19 wavenumbers in the spectral range of $1700-5000 \mathrm{~cm}^{-1}(2-6$ $\mu \mathrm{m})$, chosen outside of strong gas absorption bands. To increase the signal-to-noise ratio, spectra are averaged using moving average over a $50-\mathrm{cm}^{-1}$ spectral window and a 2-km altitude window; the number of averaged spectra depends on the altitude sampling and typically equals 400-800.

The procedure to obtain transmittances from the TIRVIM dataset is straightforward. This channel is operated continuously and therefore remains stable during an occultation. Slant optical depth $\tau$ is calculated as $\tau(L)=-\ln (\operatorname{Tr}(L))$, where $\operatorname{Tr}$ is the transmittance over the line of sight $L$. Vertical profiles of extinction and corresponding uncertainties are retrieved using a standard “onion peeling” method (18). Full retrieval of aerosol parameters from TIRVIM extinction profiles involves the fitting of the experimental data using Mie modeling to obtain vertical profiles of the size distribution and number density. Before model fitting, we normalized all obtained extinction coefficients by the extinction at the wavelength with the lowest noise. For the Mie modeling, we used water ice optical constants in the temperature range of $130-210 \mathrm{~K}(46)$, at $266 \mathrm{~K}$ (47), and optical constants of the mineral dust (48). We used temperature profiles from NIR channel (see above) or MCD5.3 (37), if NIR data is unavailable. We assumed a log-normal law to model the size distribution of particles (49). Forward modeling of extinction crosssections results in two look-up tables for each of the aerosol types, and for a set of effective radii $r_{\text {eff, }}$, effective variances $v_{\text {eff, }}$, and temperatures (in the case of water ice).

To distinguish between the water ice and dust particles, we apply the LevenbergMarquardt retrieval scheme independently for either type, or the bimodal case, and make a decision based on the fit quality. The derived free parameters are the effective radius of a single-mode in the unimodal case and effective radius of both modes and the ratio of their number densities $\gamma$ in the bimodal case. Effective variances (ranging within 0.1-0.6 with 0.1 steps) are not fitted directly, but determined from the best estimate brute-force search, which also gives the first guess of the retrieved parameter(s). Uncertainties of $r_{\text {eff }}$ and $\gamma$ are estimated from the covariance matrix. After a satisfactory fit is found, the number density $n$ is found by dividing the normalized extinction by the retrieved extinction cross-section. An example of spectral model fitting at an altitude $26 \mathrm{~km}$ of orbit 2295 is shown in Figure S11, demonstrating the presence of both the dust and water ice aerosols.

The resulting number density profiles of particles with known radii are recomputed into mass loading profiles as shown in Figure S12, assuming a density of $1 \mathrm{~g} \mathrm{~cm}^{-3}$ for the ice particles and $2.5 \mathrm{~g} \mathrm{~cm}^{-3}$ for the dust particles. 
There were several gaps in the observations performed by TIRVIM, the longest one occurred from 15 July to 1 September $2018\left(L_{s} 212^{\circ}-242^{\circ}\right)$. To increase temporal coverage of observations, we use a simplified analysis of aerosol content and incorporate MIR extinction data in the analysis. We use extinction at the center of the water ice absorption band $(\sim 3.1 \mu \mathrm{m})$ as a substitute for aerosol mass loading and extinction at several other wavelengths to distinguish between water ice and dust particles, as discussed below.

For TIRVIM, we use extinction coefficients at two of 19 wavenumbers, at $3263 \mathrm{~cm}^{-1}$ (3.06 $\mu \mathrm{m}$, ext $^{\mathrm{T}}{ }_{\mathrm{c}}$ ) and at $3446 \mathrm{~cm}^{-1}\left(2.9 \mu \mathrm{m}\right.$, ext ${ }_{\mathrm{w}}^{\mathrm{T}}$ ), as shown in Figure S11. Here and throughout, superscripts specify the channel: T, M, and N correspond to TIRVIM, MIR, and NIR. Subscripts, where present, specify the position of the wavelength inside the water ice band: $\mathrm{c}$ and $\mathrm{w}$ correspond to the center or to the wing of the absorption band. These two wavelengths allow for the decision on the aerosol type using a simple criterion based on the ratio of these extinctions $r a t^{T}=e{ }^{\mathrm{T}}{ }^{\mathrm{T}}$ dext ${ }_{\mathrm{w}}^{\mathrm{T}}$. Figure S13A shows rat ${ }^{\mathrm{T}}$ calculated from Mie theory for mineral dust and water ice at $130 \mathrm{~K}$ and $266 \mathrm{~K}$ for a $r_{\text {eff }}=$ $0.1-5 \mu \mathrm{m}$ and $v_{\text {eff }}=0.3$. Water ice is assumed if $\mathrm{rat}^{\mathrm{T}}>1.2($ area $\alpha)$; dust otherwise (area $\beta$ ). In the case of NIR, 450 spectral elements in several lines (from 6 to 20, depending on occultation) were averaged.

The same criterion could work with MIR data in the secondary grating position 13 (3150-3585 $\mathrm{cm}^{-1}$, Figure S11) (17). Unfortunately, such measurements were only obtained at the beginning of the mission when the TIRVIM dataset was very dense; therefore we discarded the position 13 data. Instead, we used extinction at $3225 \mathrm{~cm}^{-1}$ from the order 192 of the position 12 (2905-3265 cm $\mathrm{c}^{-1}$, Figure S11). Position 12 includes the methane absorption bands and was regularly used throughout the mission. However, as explained below, the absorption measured at the longer wavelength side of the $\mathrm{H}_{2} \mathrm{O}$ ice band is less informative and implies a more complicated criterion to select the aerosol type, involving also data at $10126 \mathrm{~cm}^{-1}(0.99 \mu \mathrm{m}$, Figure S11) from NIR order 78. The uncertainties of the transmission propagating into the extinction for MIR are estimated from averaging of 150 spectral elements corresponding to the central part of the detector.

The criterion to decide on the aerosol type is based on two values: the ratio of MIR extinctions at $3225 \mathrm{~cm}^{-1}\left(e x t^{\mathrm{M}}{ }_{C}\right)$ and at $2905 \mathrm{~cm}^{-1}\left(e x t^{\mathrm{M}}{ }_{\mathrm{w}}\right) r a t^{\mathrm{M}}=e^{-1 x t^{\mathrm{M}}}{ }_{c}{ }^{e x t^{\mathrm{M}}}{ }_{\mathrm{w}}$, and the ratio of ext ${ }_{\mathrm{w}}^{\mathrm{M}}$ and the NIR extinction at $10126 \mathrm{~cm}^{-1}\left(e^{-1} t^{\mathrm{N}}\right) \operatorname{rat}^{\mathrm{MN}}=e x \mathrm{t}_{\mathrm{w}}^{\mathrm{M}}{ } / \mathrm{ext}^{\mathrm{N}}$. The dependence of $r a t^{\mathrm{T}}, r a t^{\mathrm{M}}$ and $r a t^{\mathrm{MN}}$ on the effective radius is shown in Figure S13.The ratio $r a t^{\mathrm{MN}}$ serves to estimate the particle size $\left(r_{\text {eff }}>1.5 \mu \mathrm{m}\right.$ in area I, and $r_{\text {eff }}<1.5 \mu \mathrm{m}$ in area II). The area III corresponds to dust particles. Comparing $\mathrm{rat}^{\mathrm{M}}$ with $\mathrm{rat}^{\mathrm{MN}}$ allows deciding on the nature of the particles. Figure S13B, the areas a, b and e correspond to water ice particles, while the areas $\mathrm{c}$ and $\mathrm{d}$ correspond to dust particles. If ext ${ }^{\mathrm{N}}$ is not available at a certain altitude $H$ (at lower altitudes the NIR channel signal vanishes), we assume that $r_{\text {eff }}$ $>1.5 \mu \mathrm{m}$ if $H<25 \mathrm{~km}$, and vice-versa. The boundaries of the described areas were tuned to keep to a minimum disagreement between the results of the extinction retrievals described above, and the approximate judgment criteria. The described criteria to decide about the aerosol type are summarized in Table S2. Some ambiguity remains in the areas c and d, where water ice particles might be erroneously labeled as dust particles (when $1.2 \mu \mathrm{m}<r_{\text {eff }}<1.8 \mu \mathrm{m}$ for water ice). This has occurred only once out of 21 coinciding TIRVIM, MIR and NIR occultations, used for the validation of this simplified procedure. The ACS MIR data provided additional aerosol loading profiles completing the dataset. 
Figure S12 shows a comparison of the extinction as a proxy of mass loading to the retrieved mass loading using an example occultation 2580 at $L_{s} 198^{\circ}$. Figure S14 shows the evolution of water ice content $\left(L_{s} 170-255^{\circ}\right.$ vs altitude) calculated from proper retrieval of aerosol mass loading (panel A) and estimated by extinction (panel B). They are in qualitative agreement, the only difference being two regions near $L_{s} 200^{\circ}$ and $210^{\circ}$ at altitudes $10-40 \mathrm{~km}$. These regions are dominated by dust, but also contain some water ice, which is not reproduced by the simplified approach due to the assumption of unimodality. 

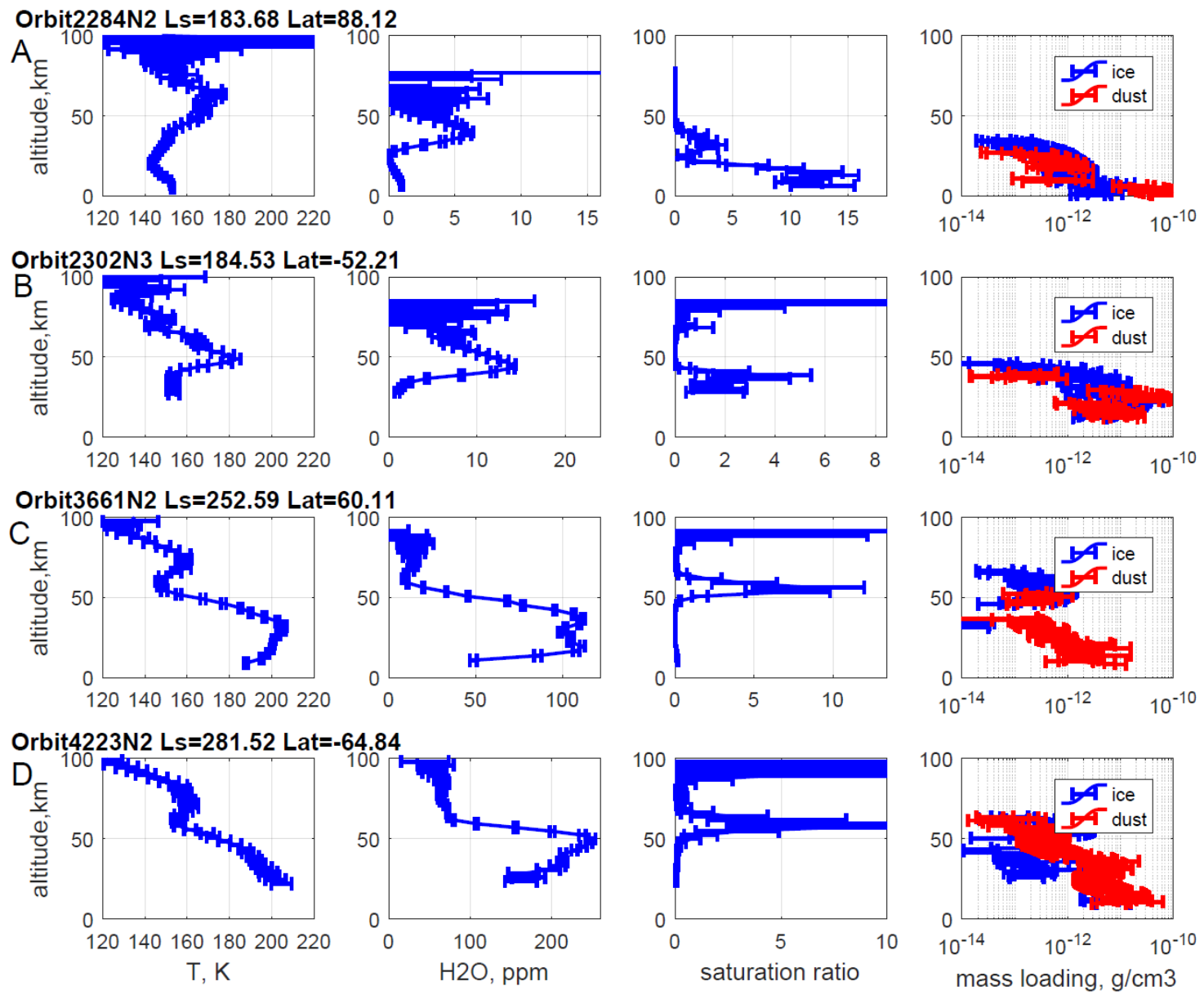

Fig. S1. Example cases of supersaturation:

Four ACS NIR occultations showing occurrences of supersaturation: temperatures, water vapor vmr profile, saturation ratio and aerosol mass loading (dust and water ice) (see also Figure 1). The altitudes are above the areoid; (A) - orbit $2284\left(L_{s}=183.68^{\circ}, 88.12^{\circ} \mathrm{N}\right.$, $46.8^{\circ} \mathrm{E}$, local time 12:20); (B) orbit $2302\left(L_{s}=184.53^{\circ}, 52.21^{\circ} \mathrm{S}, 143.9^{\circ} \mathrm{E}\right.$, local time 05:50); (C) orbit $3661\left(L_{s}=253.59^{\circ}, 60.11^{\circ} \mathrm{N}, 152.5^{\circ} \mathrm{W}\right.$, local time 14:35); (D) orbit $4223\left(L_{s}=281.52^{\circ}, 64.84^{\circ} \mathrm{S}, 101.42^{\circ} \mathrm{E}\right.$, local time $\left.00: 34\right)$ 

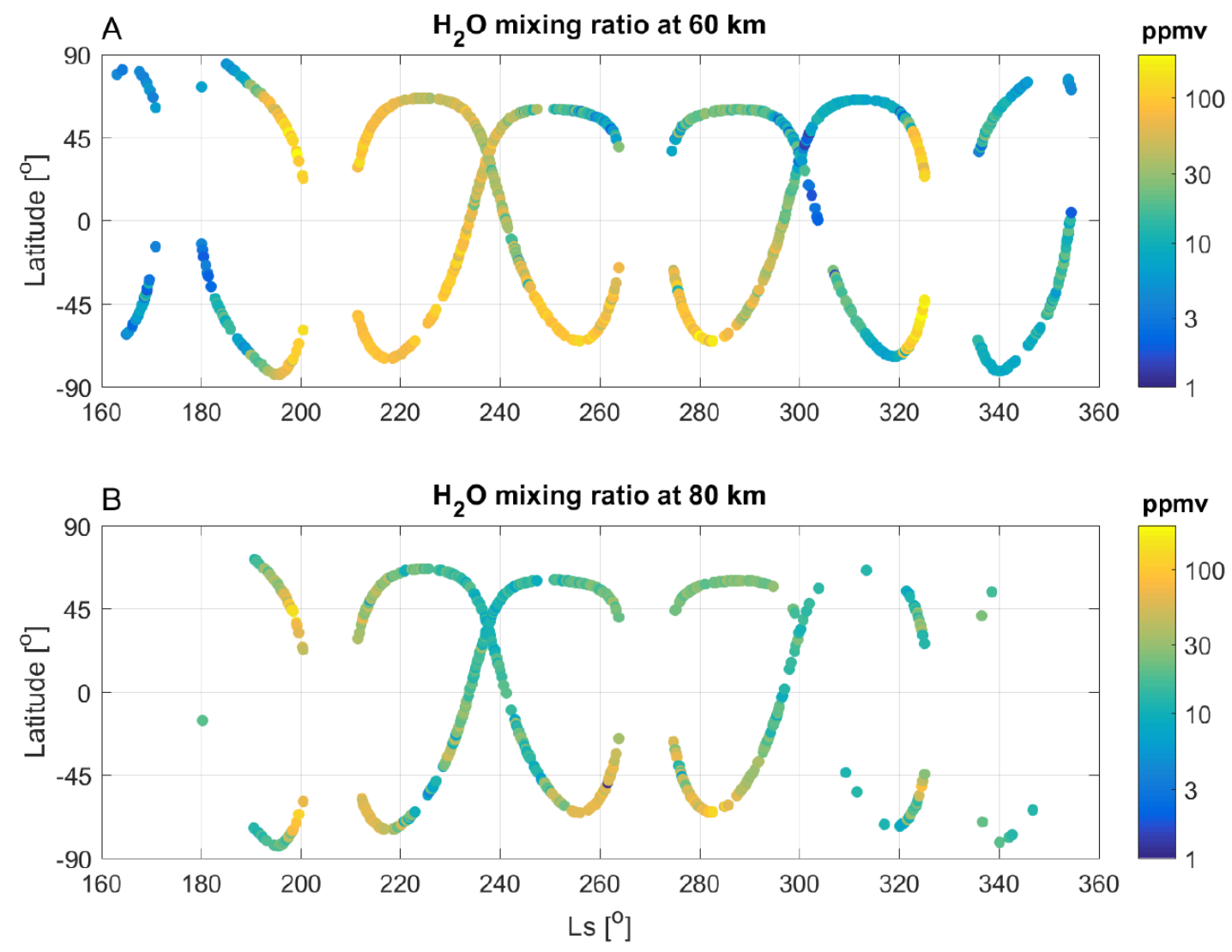

Fig. S2. High altitude water:

Seasonal-latitudinal distribution of water vapor mixing ratio in ppmv at $60 \mathrm{~km}$ (A) and 80 $\mathrm{km}(\mathrm{B})$. 


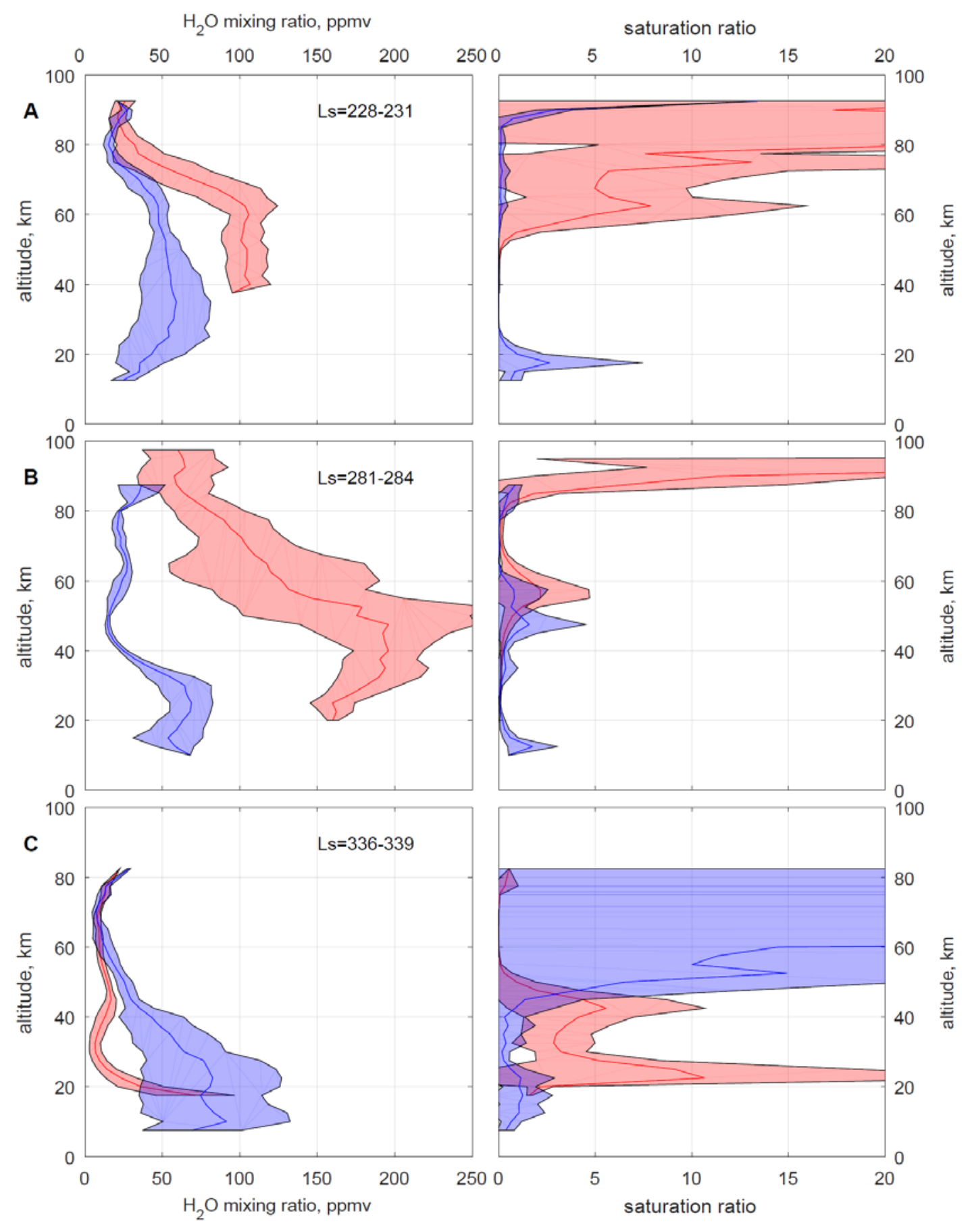

Fig. S3: Binned profiles of water vapor and saturation ratio:

A different perspective of Figure 2, superimposing the averaged vmrs and saturation ratio profiles in the northern (blue) and southern (red) hemispheres binned into three characteristic periods encompassing the second half of MY34: (A) the decay of 2018 GDS; (B) the perihelion period of the southern summer; (C) end of the regional dust storm $C$; The shaded areas indicate the statistical dispersion $(1 \sigma)$ of measurements. 

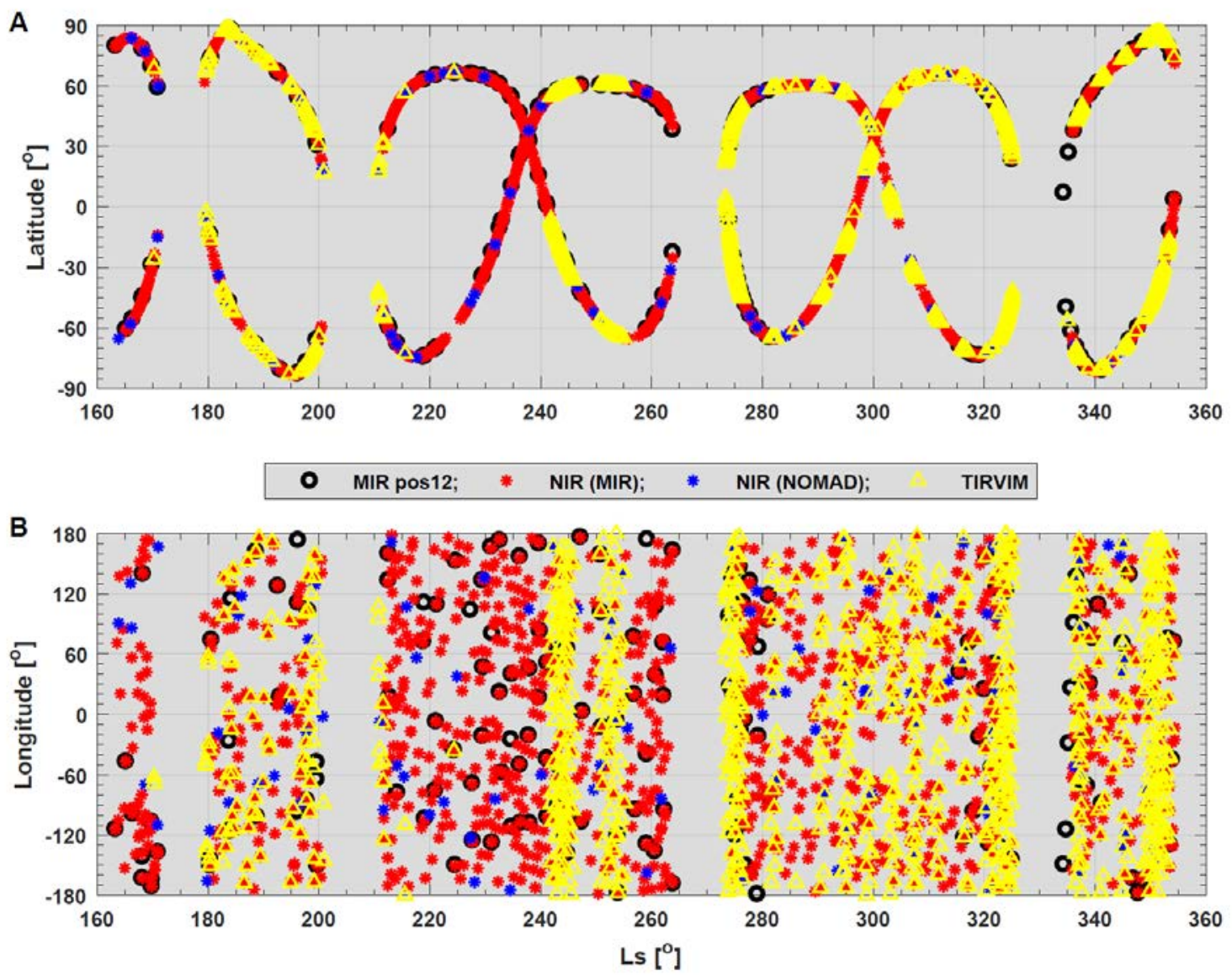

Fig. S4: Overview of ACS measurements used in this study:

(A) latitude evolution of occultations; (B) the longitudinal spread. NIR occultations (asterisks) are depicted by different colors for MIR (red) and NOMAD (blue) pointings. MIR (black circles), and TIRVIM (yellow triangles) occultations are also shown. The coordinates of the occultations are given as mean values at the reference altitude range 0 $100 \mathrm{~km}$. 

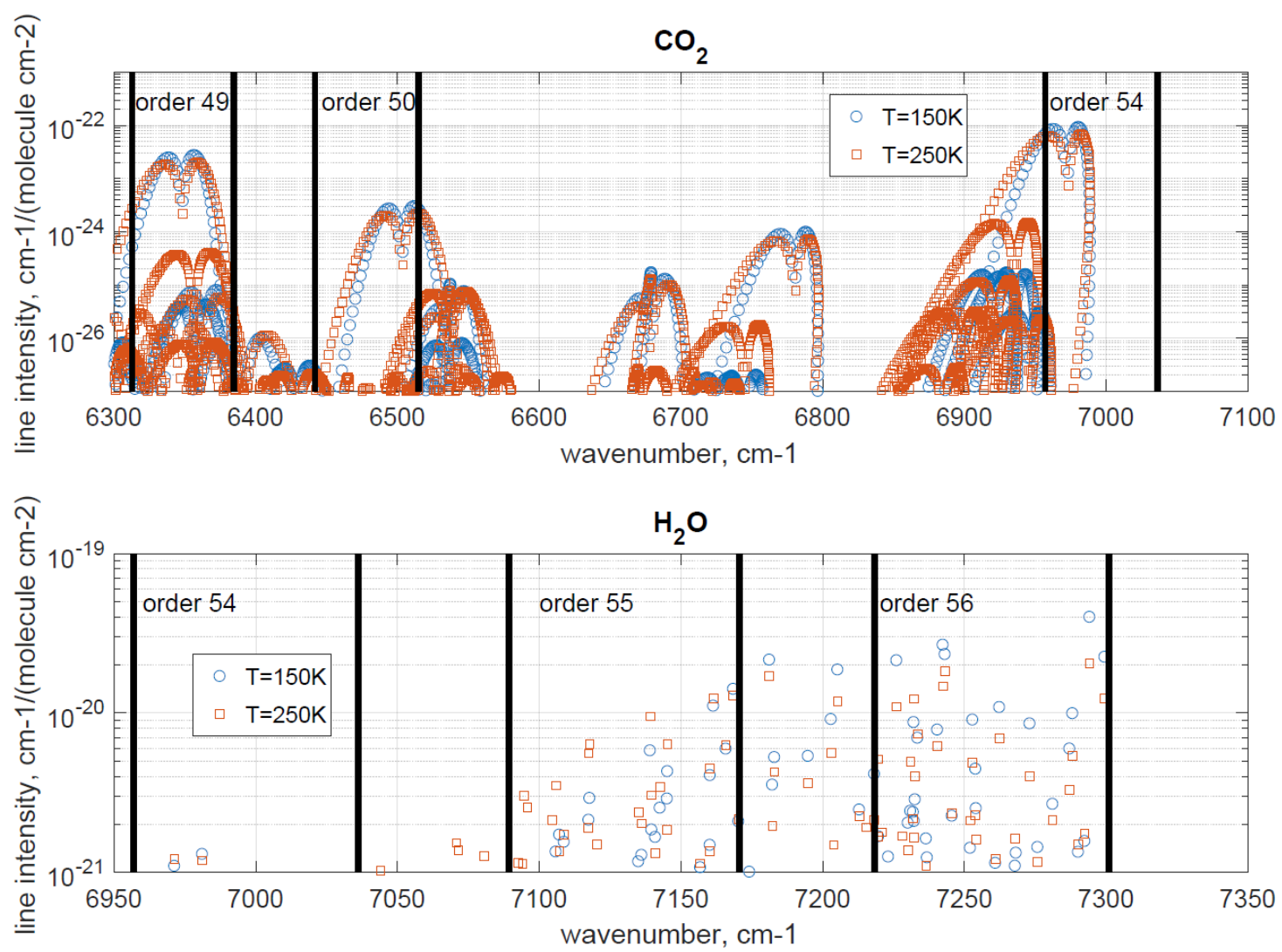

Fig.S5: Sensitivity of near-IR molecular lines to temperature following HITRAN 2016 (32)

(A) $\mathrm{CO}_{2}$ lines, (B) $\mathrm{H}_{2} \mathrm{O}$ lines. The bounds of the ACS NIR spectral ranges (diffraction orders), as measured in a standard occultation sequence, are indicated by vertical bars. 


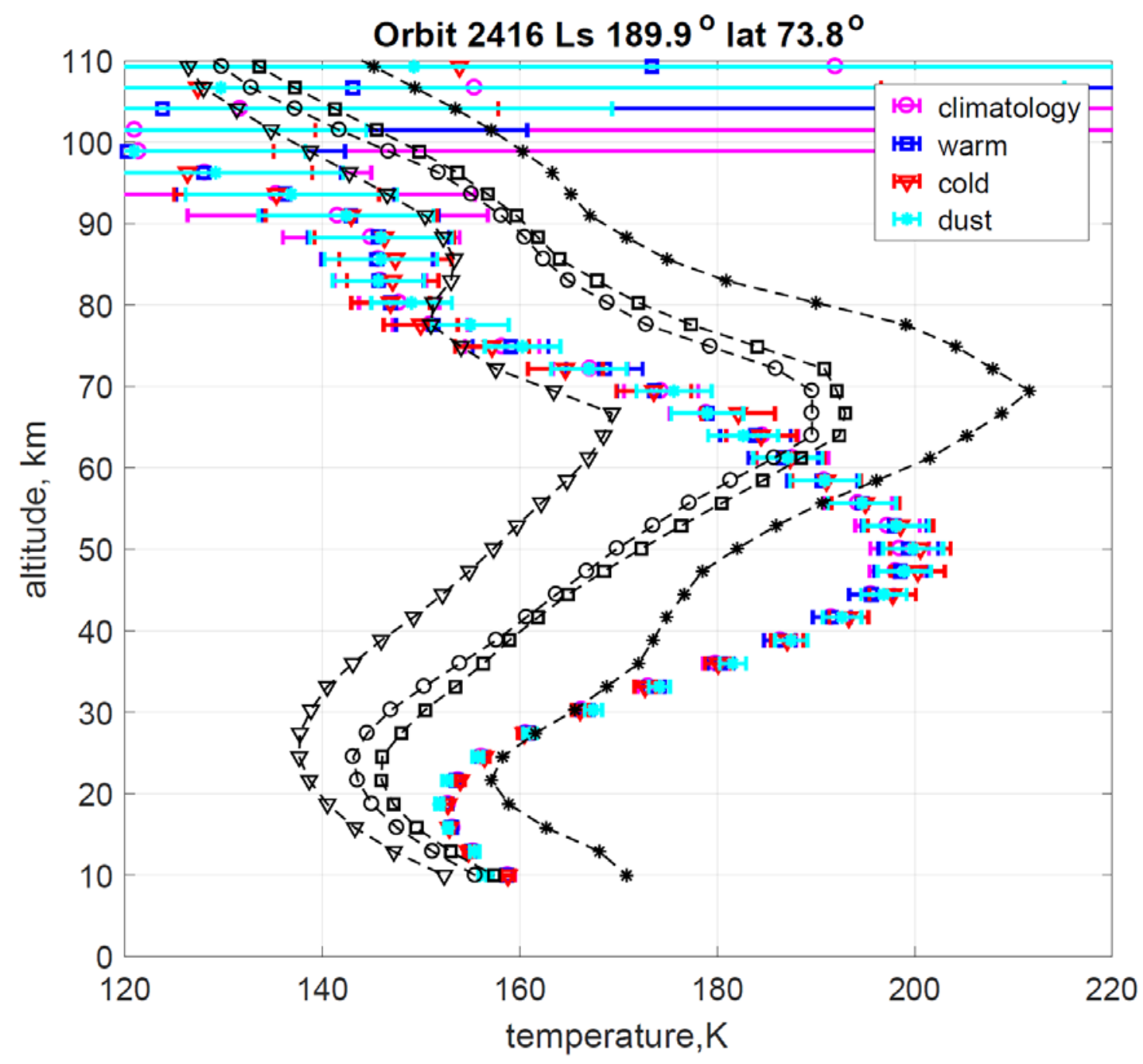

Fig.S6: Sensitivity of the temperature retrieval to the initial assumption.

An example of retrievals with four different MCD scenarios (climatology, dust, warm and cold) as an initial assumption (black dashed lines). The resulting profiles are shown as color curves. The error bars (one-sigma) are given by the retrieved covariance matrix. 

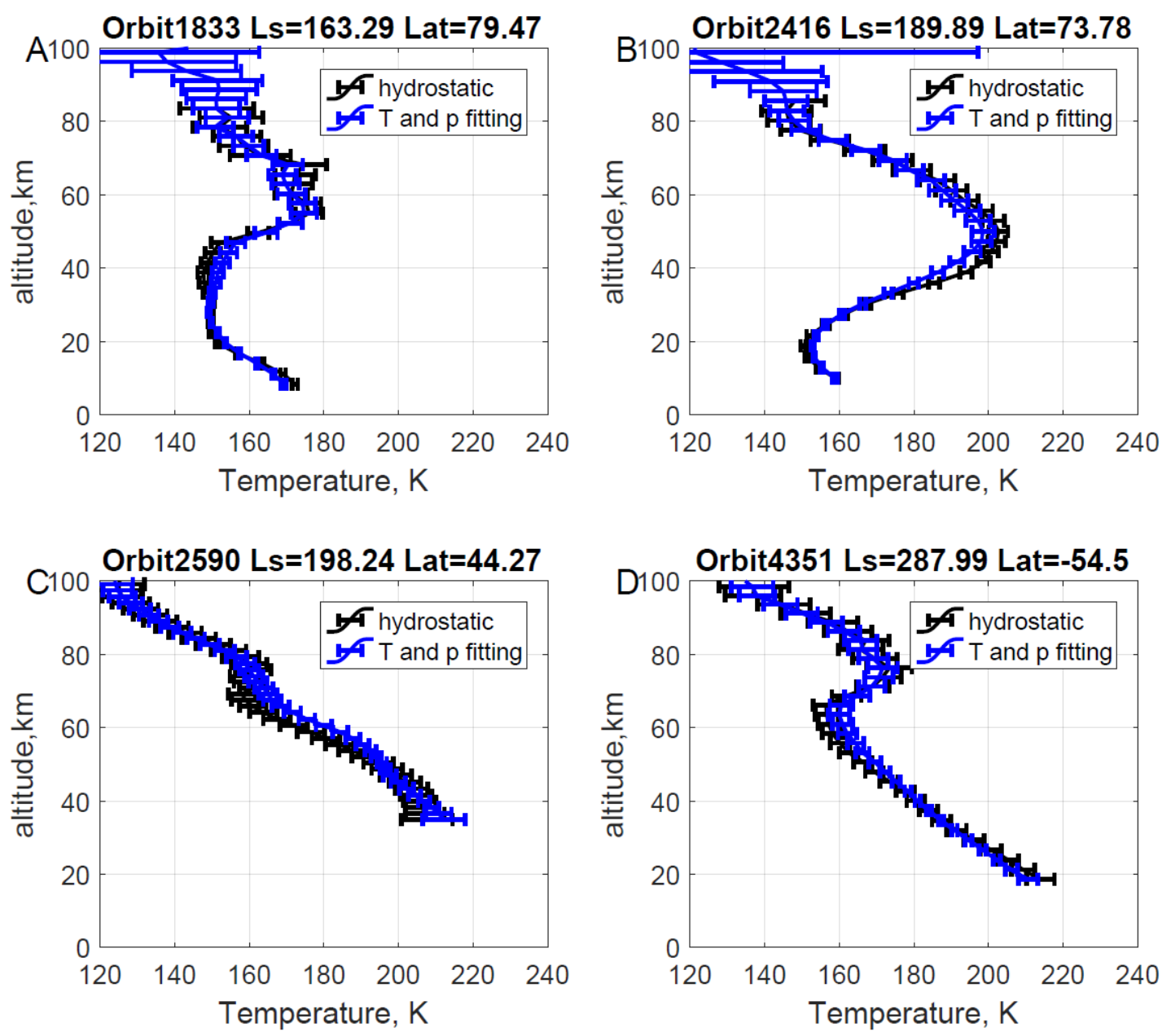

Fig. S7: The comparison of hydrostatic temperature with independent $T$ and $P$ retrieval.

Examples for four different orbits: (A) - orbit $1833\left(L_{s}=163.8^{\circ}, 79.5^{\circ} \mathrm{N}, 114.0^{\circ} \mathrm{W}\right.$, local time 03:26); (B) orbit $2416\left(L_{s}=189.9^{\circ}, 73.8^{\circ} \mathrm{S}, 67.6^{\circ} \mathrm{W}\right.$, local time 17:01); (C) orbit $2590\left(L_{s}=198.2^{\circ}, 44.3^{\circ} \mathrm{N}, 11.4^{\circ} \mathrm{W}\right.$, local time 17:30); (D) orbit $4351\left(L_{s}=288.0^{\circ}, 54.5^{\circ} \mathrm{S}\right.$, $27.4^{\circ} \mathrm{W}$, local time 20:35). The error bars (one-sigma) are given by the retrieved covariance matrix. 

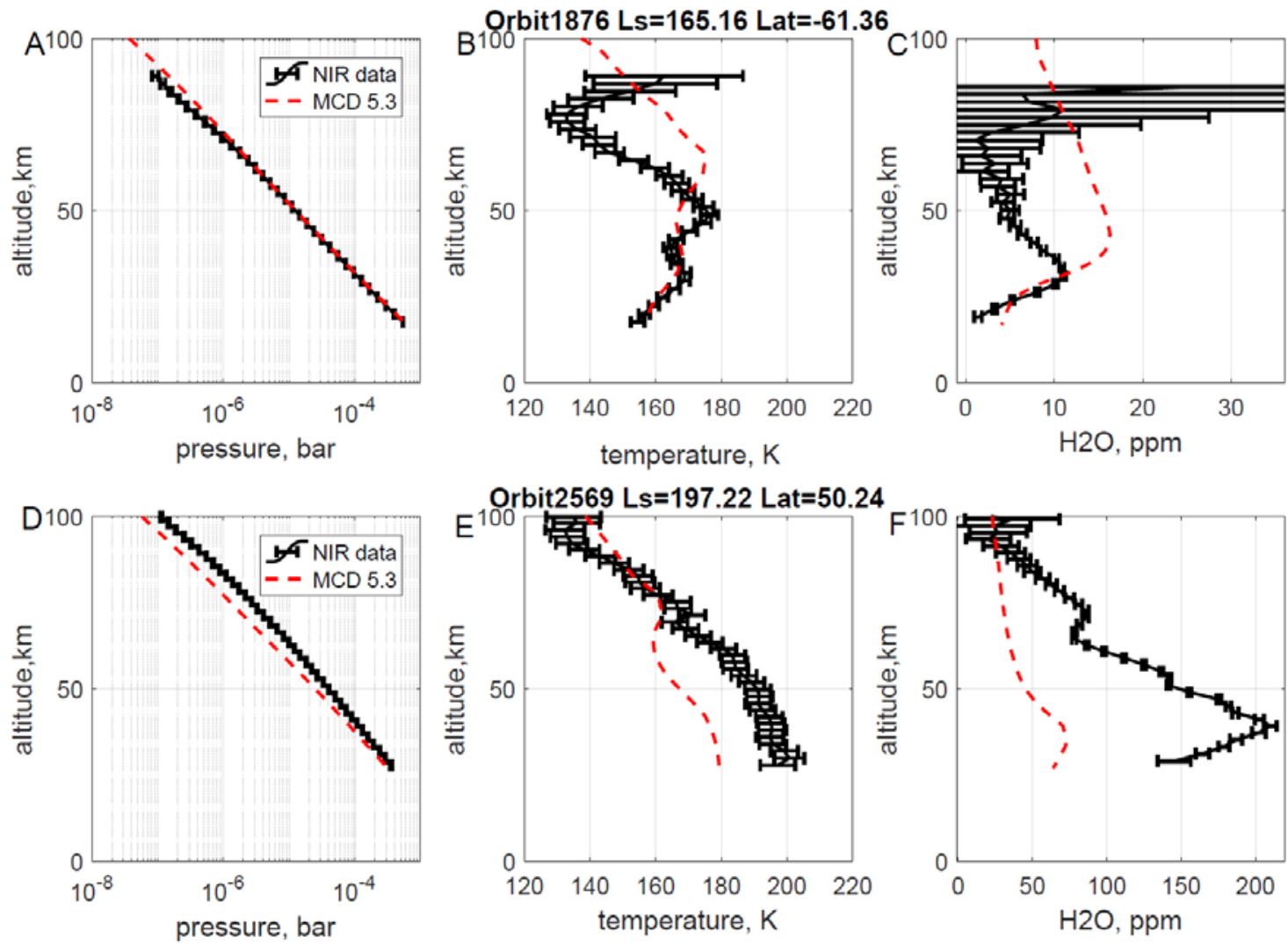

Fig. S8: Two examples of ACS NIR retrievals.

A and D panels: pressure, derived from the $\mathrm{CO}_{2}$ number density; $\mathrm{B}$ and $\mathrm{E}$ panels: atmospheric temperature; $\mathrm{C}$ and $\mathrm{F}$ panels: $\mathrm{H}_{2} \mathrm{O}$ profiles (black lines), compared to MCD5.3 "climatology" (37) prediction (dashed red line). The measurements in orbits 1876 and 2569 occurred before the global dust storm and during the storm, respectively. The error bars (one-sigma) are given by the retrieved covariance matrix. 
Orbit $2233-$ Lat $=-25.91-$ Lon $=-33.42-$ Ls $=181.32$
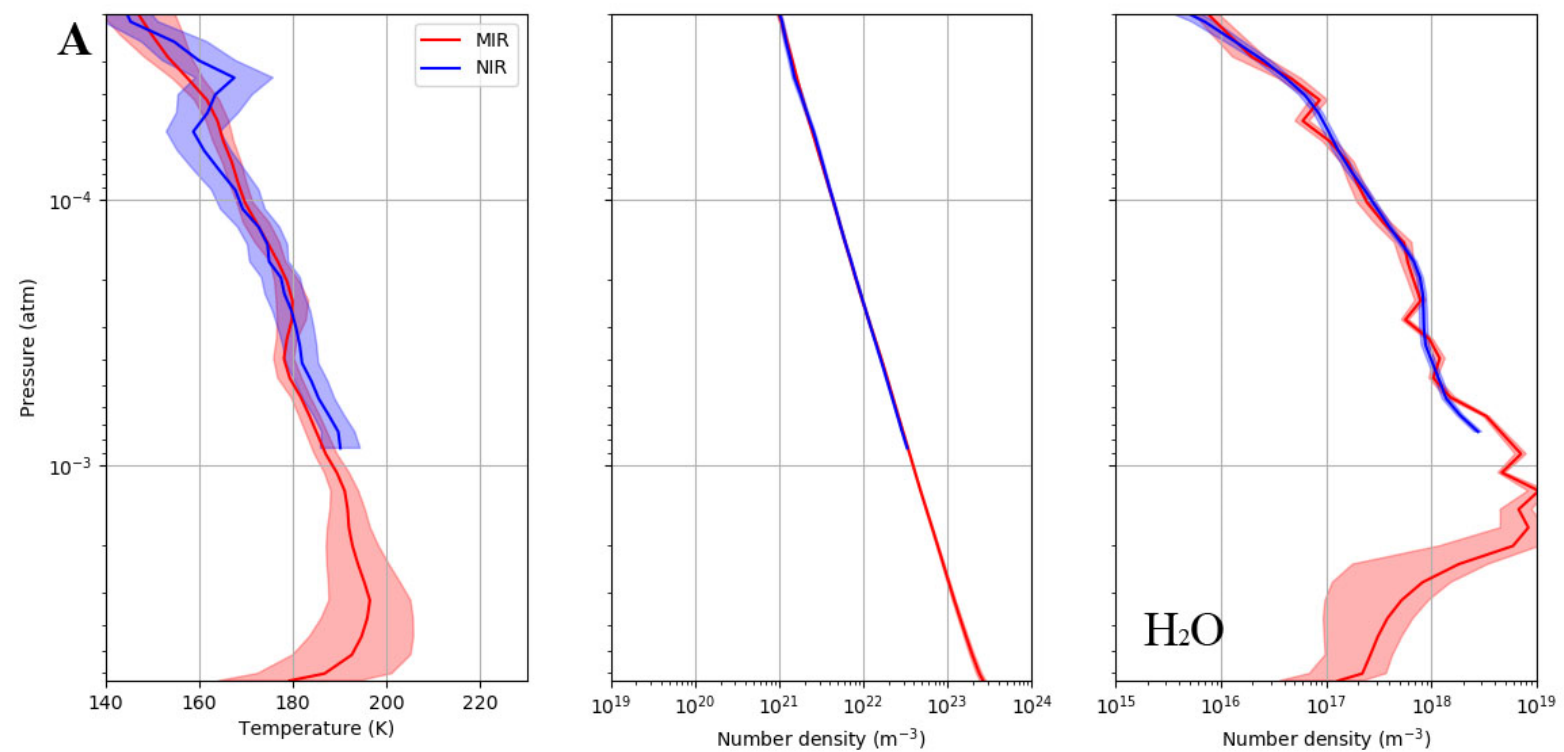

Orbit $2892-$ Lat $=42.53-$ Lon $=177.59-$ Ls $=213.11$
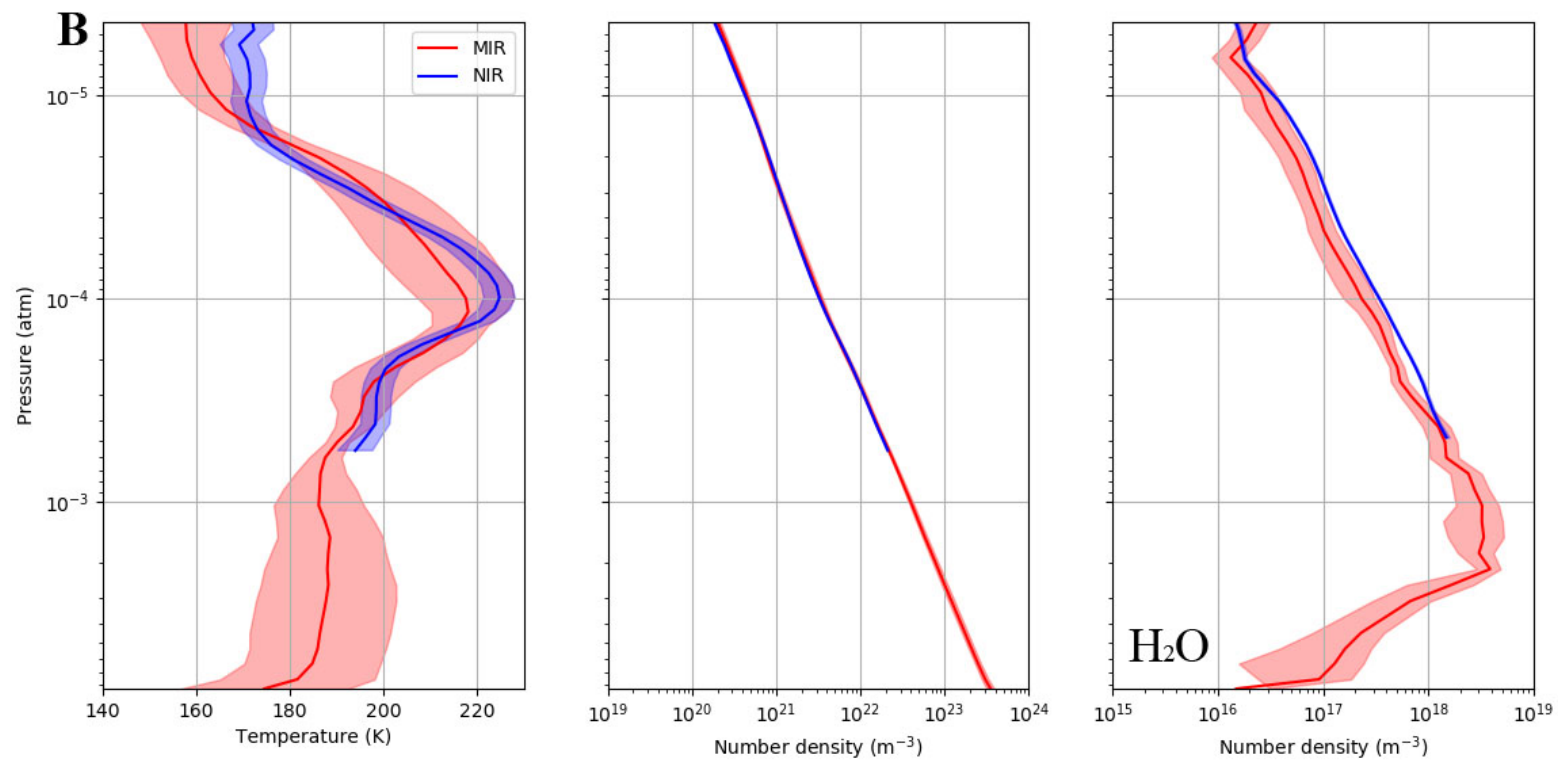

Fig. S9: Cross-validation of the atmospheric profiles retrieved from ACS NIR and ACS MIR

Two example cases are shown: before the global dust storm (A - orbit $2233\left(L_{s}=181.32^{\circ}\right.$, latitude of $-25.9^{\circ}$, longitude of $33.42^{\circ}$, local time $05: 58$ ) and during the storm (B - orbit $2892\left(L_{s}=213.11^{\circ}\right.$, latitude of $42.5^{\circ}$, longitude of $177.6^{\circ}$, local time $\left.06: 54\right)$. The shaded areas are uncertainties (one-sigma) given by the retrieved covariance matrix. 

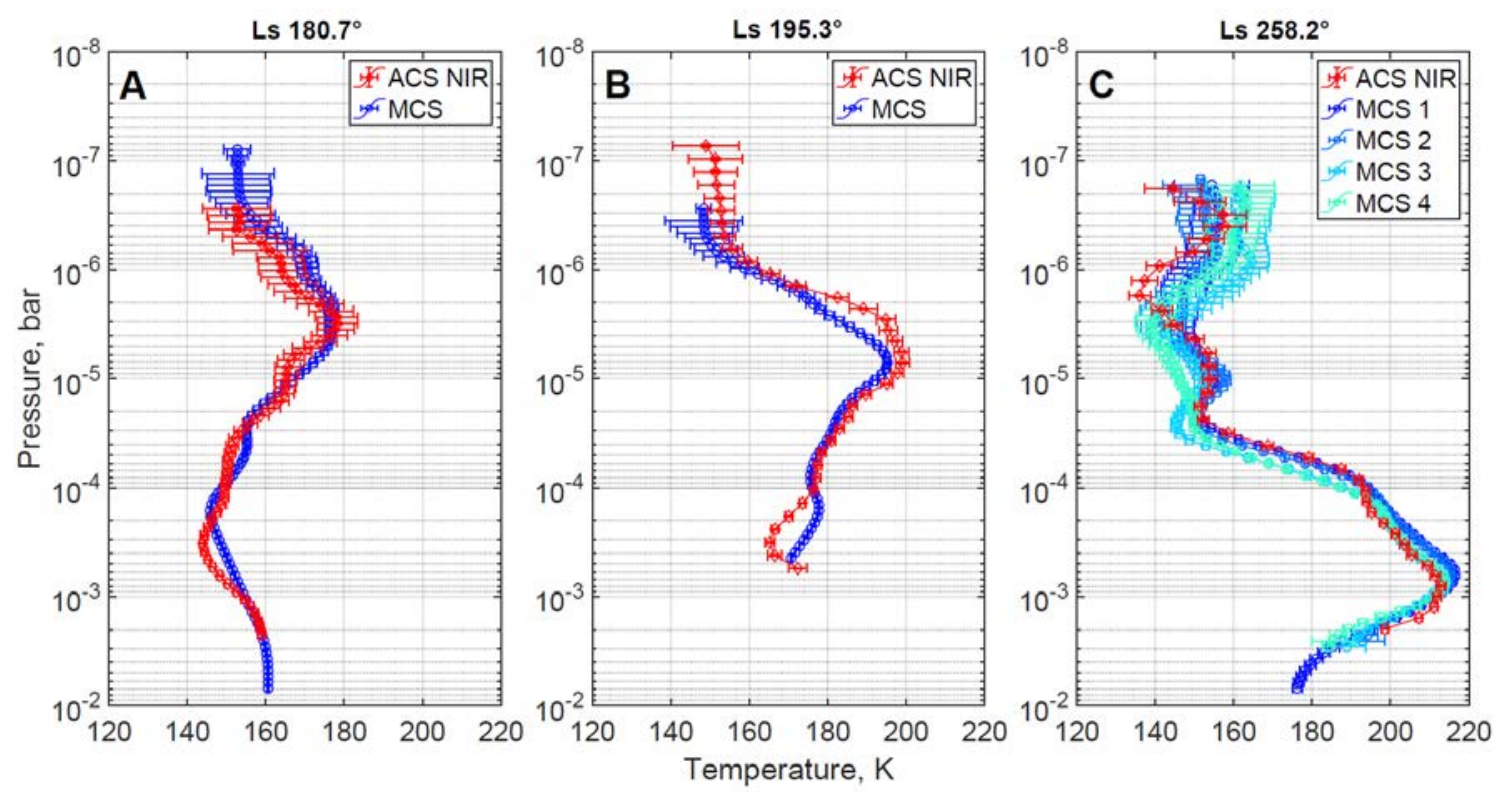

Fig. S10: Cross-validation of ACS NIR and MCS/MRO

The atmospheric profiles retrieved from ACS NIR (red points) and MCS/MRO (blue and cyan points) for three example cases listed in Table S3. 
Orbit \#2295, Altitude 26.0 km, Ls 184.2, Lat -51, Lon -15, Local Time 6 h

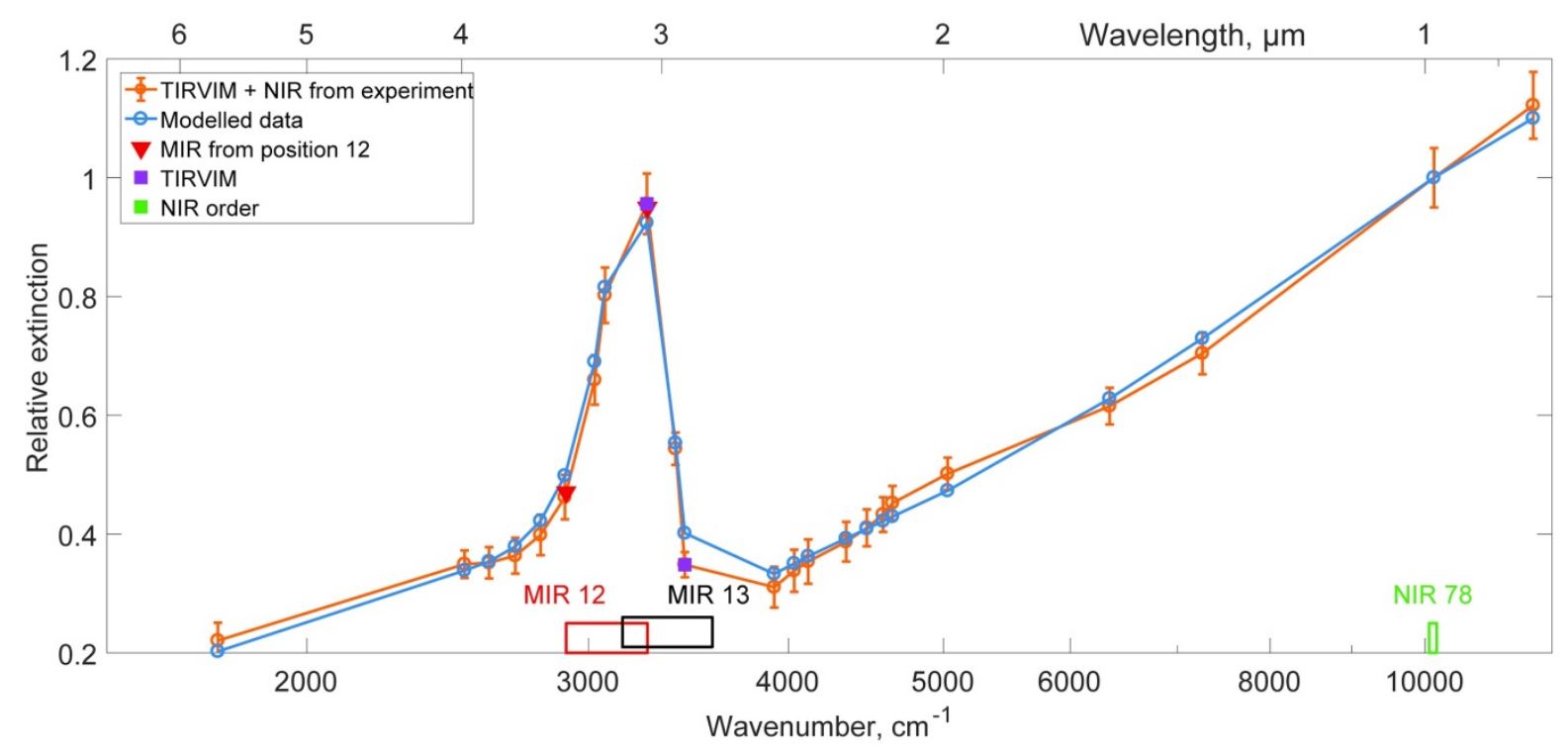

Fig. S11: Aerosol extinction as measured by ACS TIRVIM, MIR and NIR channels. Example measurement at $26 \mathrm{~km}$ altitude in orbit 2295 is shown. Extinctions are normalized to the TIRVIM value at $1 \mu \mathrm{m}$. Mie model (blue curve) is fitted to experimental data (orange curve with error bars), yielding $r_{\text {eff }}=3.1 \pm 0.6 \mu \mathrm{m}$, $n=0.08 \pm 0.02 \mathrm{~cm}^{-3}$, and $v_{\text {eff }}=0.6$ for dust particles and $r_{\text {eff }}=0.7 \pm 0.1 \mu \mathrm{m}$, $n=3.2 \pm 0.7 \mathrm{~cm}^{-3}$, and $v_{\text {eff }}=0.3$ for water ice particles. Purple squares: TIRVIM extinctions at $3263 \mathrm{~cm}^{-1}$ and $3446 \mathrm{~cm}^{-1}$ used for the analysis of aerosol content. Red and black rectangles: the spectral range of MIR positions 12 and 13. Red triangles: MIR extinctions at $3225 \mathrm{~cm}^{-1}$ and $2905 \mathrm{~cm}^{-1}$. Green rectangle: the spectral range of NIR order 78. Green square: NIR extinctions at $10126 \mathrm{~cm}^{-1}$. 

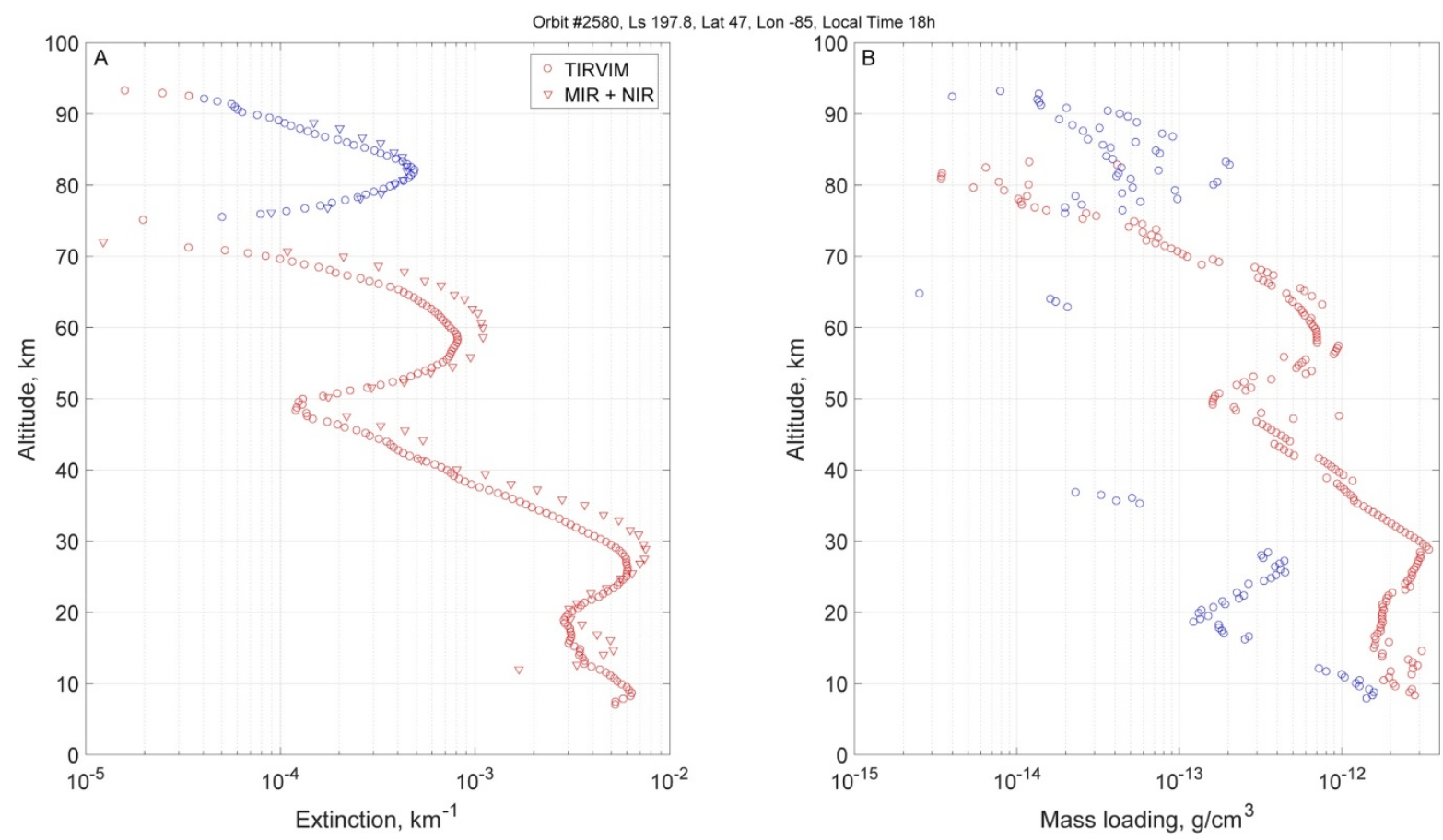

Fig. S12: Extinction and mass loading profiles

Profiles of dust and ice aerosol extinction from TIRVIM (circles) and MIR (triangles) for an example occultation 2580 at $L_{s}=198^{\circ}$ (panel A) against the aerosol mass loading (panel B) retrieved as explained in the text, showing the validity of the extinction as a proxy for the mass loading. Water ice particles are shown in blue, dust is shown in red. Error bars are omitted for clarity (cf. Fig. 1). 

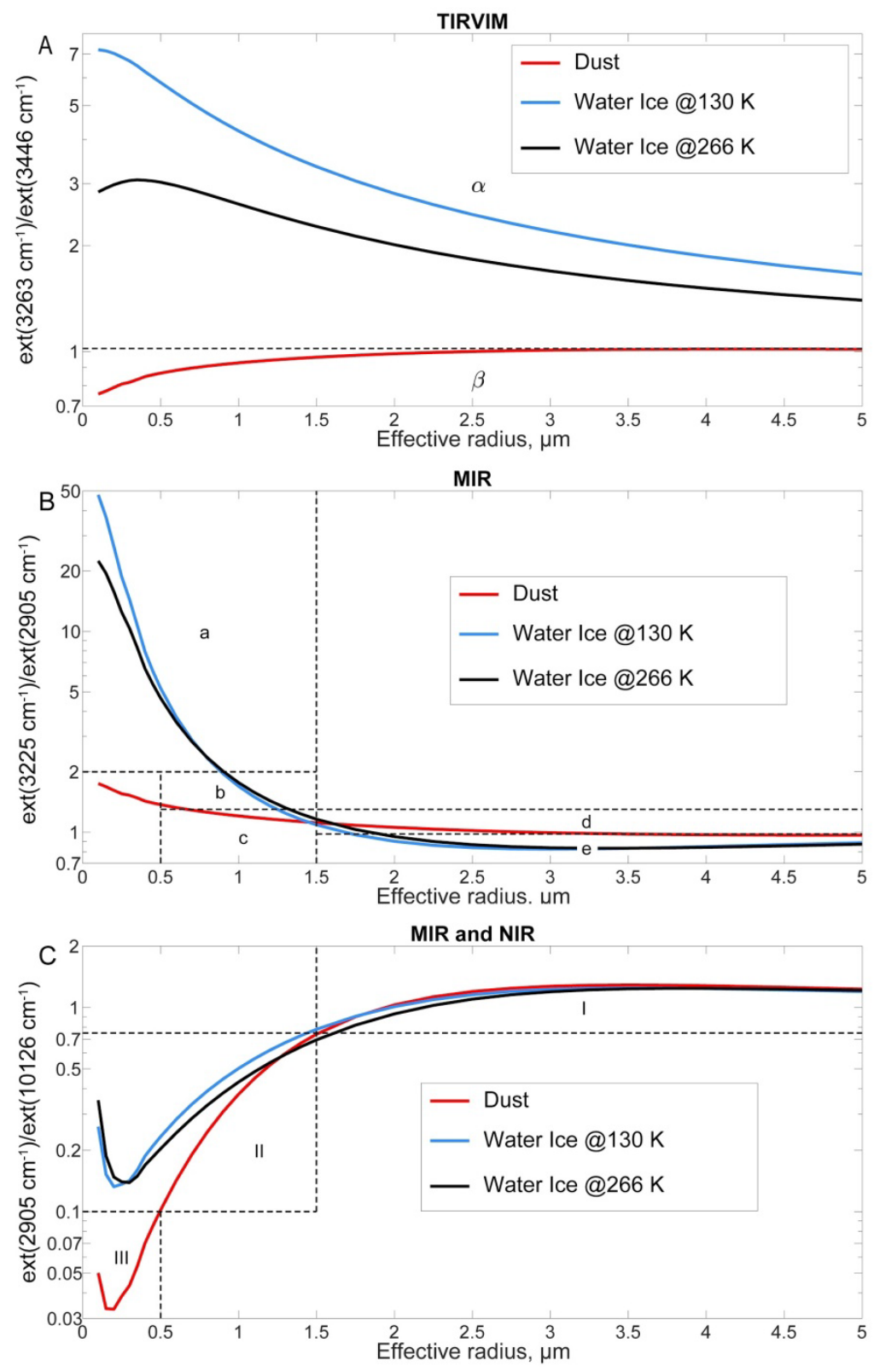

Fig. S13: Mapping of aerosol particles in the space of extinctions at different wavelengths vs effective radius.

Panel A: ratio of TIRVIM extinctions at $3263 \mathrm{~cm}^{-1}$ and $3446 \mathrm{~cm}^{-1}$ calculated from Mie theory for mineral dust (red) and water ice at $130 \mathrm{~K}$ (blue) and $266 \mathrm{~K}$ (green) for a $r_{\text {eff }}=$ $0.1-5 \mu \mathrm{m}$ and $v_{\text {eff }}=0.3$. $\alpha$ and $\beta$ regions correspond to water ice and dust aerosol types respectively. Panel B: the ratio of MIR extinctions at $3225 \mathrm{~cm}^{-1}$ and $2905 \mathrm{~cm}^{-1}$ for the same conditions as in panel A. Areas a, b, and e are indicative of water ice particles; c and d correspond to dust particles. Panel C: the ratio of MIR extinction at $2905 \mathrm{~cm}^{-1}$ and NIR extinction at $10126 \mathrm{~cm}^{-1}$ for the same conditions as in panel A. The areas I and II correspond to particles with $r_{\text {eff }}>1.5 \mu \mathrm{m}$ and $r_{\text {eff }}<1.5 \mu \mathrm{m}$ respectively; area III corresponds to dust particles. 

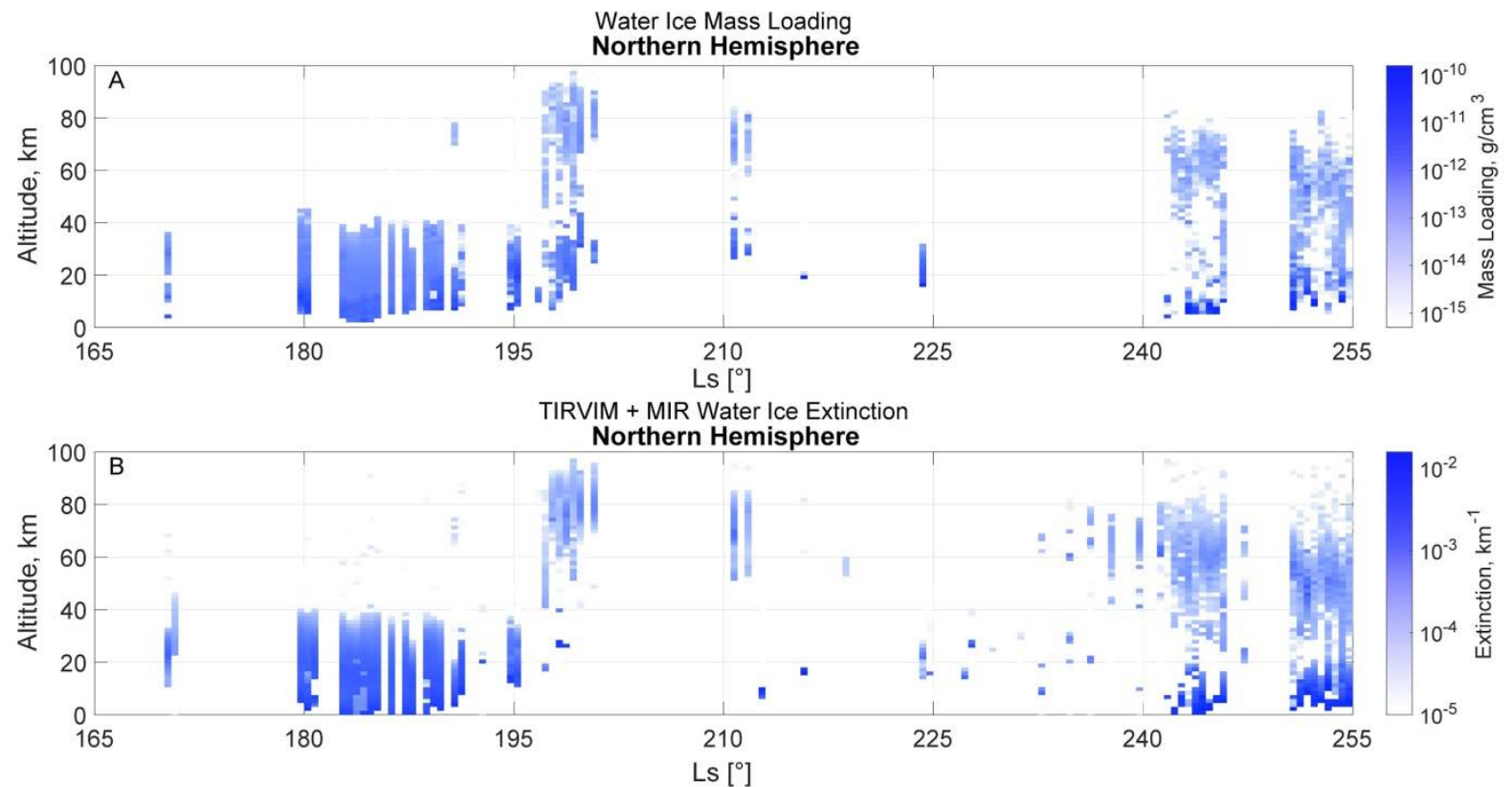

Fig. S14: Water ice distribution with season

Evolution of water ice content $\left(L_{s} 170-255^{\circ}\right.$ vs altitude) calculated from the retrieval of aerosol mass loading (panel A) and estimated by extinction (panel B). 
Table S1 Number of occultation profiles by different ACS channels used in this study.

\begin{tabular}{|c|c|c|c|}
\hline \multicolumn{2}{|c|}{ NIR } & \multirow{2}{*}{$\begin{array}{c}\text { MIR } \\
\text { (the secondary grating } \\
\text { position 12) }\end{array}$} & \multirow{2}{*}{$\begin{array}{c}\text { TIRVIM } \\
\text { (climatology mode) }\end{array}$} \\
\hline MIR pointing & $\begin{array}{l}\text { NOMAD } \\
\text { pointing }\end{array}$ & & \\
\hline 1143 & 110 & 161 & 889 \\
\hline
\end{tabular}


Table S2. Criteria for aerosol type selection.

\begin{tabular}{|c|c|c|c|c|}
\hline & \multicolumn{2}{|l|}{ Water ice } & \multicolumn{2}{|l|}{ Dust } \\
\hline & Criterion & Area & Criterion & Area \\
\hline TIRVIM & $\mathrm{rat}^{\mathrm{T}}>1.2$ & $\alpha$ & $\mathrm{rat}^{\mathrm{T}}<1.2$ & $\beta$ \\
\hline \multirow[t]{3}{*}{ MIR and NIR } & $r a t^{\mathrm{MN}}<0.1$ & III & - & - \\
\hline & $\begin{array}{c}0.1<r a t^{\mathrm{MN}}<0.75 \\
\mathrm{rat}^{\mathrm{M}}>1.3\end{array}$ & $\begin{array}{l}\text { II a } \\
\text { II b }\end{array}$ & $\begin{array}{c}0.1<r a t^{\mathrm{MN}}<0.75 \\
\operatorname{rat}^{\mathrm{M}}<1.3\end{array}$ & II C \\
\hline & $\begin{aligned} r a t^{\mathrm{MN}} & >0.75 \\
r a t^{\mathrm{M}} & <0.9\end{aligned}$ & I e & $\begin{aligned} r a t^{\mathrm{MN}} & >0.75 \\
r a t^{\mathrm{M}} & >0.9\end{aligned}$ & I d \\
\hline
\end{tabular}


Table S3. List of observations in Fig. S7.

\begin{tabular}{|c|c|c|c|c|}
\hline Profile & $\begin{array}{c}L_{s} \\
\text { (deg) }\end{array}$ & $\begin{array}{l}\text { Latitude } \\
\text { (deg) }\end{array}$ & $\begin{array}{l}\text { Longitude } \\
\text { (deg) }\end{array}$ & $\begin{array}{l}\text { Local time } \\
\text { (h) }\end{array}$ \\
\hline \multicolumn{5}{|c|}{ (a) Before the 2018 GDS } \\
\hline ACS NIR & 180.8 & 76.9 & -40.6 & 6.1 \\
\hline MCS & 180.7 & 77.8 & -41.0 & 4.4 \\
\hline \multicolumn{5}{|c|}{ (b) During the 2018 GDS } \\
\hline ACS NIR & 195.3 & 58.4 & -93.3 & 17.3 \\
\hline MCS & 195.4 & 58.4 & -92.9 & 18.1 \\
\hline \multicolumn{5}{|c|}{ (c) After the 2018 GDS } \\
\hline ACS NIR & 258.3 & 57.4 & -32.2 & 14.9 \\
\hline MCS 1 & 258.2 & 56.5 & -31.3 & 14.6 \\
\hline MCS 2 & 258.2 & 57.0 & -31.5 & 14.5 \\
\hline MCS 3 & 258.2 & 57.5 & -31.6 & 14.5 \\
\hline MCS 4 & 258.2 & 58.1 & -31.7 & 14.5 \\
\hline
\end{tabular}

